\title{
Vector meson production from a polarized nucleon
}

\author{
M. Diehl \\ Deutsches Elektronen-Synchroton DESY, 22603 Hamburg, Germany
}

\begin{abstract}
We provide a framework to analyze the electroproduction process $e p \rightarrow e p \rho$ with a polarized target, writing the angular distribution of the $\rho$ decay products in terms of spin density matrix elements that parameterize the hadronic subprocess $\gamma^{*} p \rightarrow \rho p$. Using the helicity basis for both photon and meson, we find a representation in which the expressions for a polarized and unpolarized target are related by simple substitution rules.
\end{abstract}

KEYwords: Lepton-Nucleon Scattering, Spin and Polarization Effects. 


\section{Contents}

1. Introduction 1

2. Kinematics and target polarization 2

3. Helicity amplitudes and spin density matrix 4

4. The angular distribution $\quad 9$

$\begin{array}{ll}\text { 5. Natural and unnatural parity } & 17\end{array}$

6. Positivity constraints 21

7. Mixing between transverse and longitudinal polarization 23

8. A note on non-resonant contributions 27

$\begin{array}{lr}\text { 9. Summary } & 28\end{array}$

\section{Introduction}

Exclusive vector meson production has long played an important role in studying the strong interaction. The seminal work $[1,2]$ has renewed interest in this process, showing that in Bjorken kinematics it provides access to generalized parton distributions and thus to a wealth of information on the structure of the proton. While most theoretical and experimental studies so far are for an unpolarized proton, the particular interest of target polarization became clear when it was pointed out that meson production on a transversely polarized target is sensitive to the nucleon helicity-flip distribution $E[3,4]$. This distribution offers unique views on the orbital angular momentum carried by partons in the proton $[5,6]$ and on the correlation between polarization and the spatial distribution of partons [7]. Whereas the corresponding polarization asymmetry in deeply virtual Compton scattering is under better theoretical control, vector meson production has the advantage of a greater sensitivity to the distribution of gluons (which in Compton scattering only enters at next-to-leading order in $\alpha_{s}$ ). This holds not only in the high-energy regime but even in a wide range of fixed-target kinematics $[8,9,10]$, where polarization measurements are feasible at existing or planned experimental facilities.

A different motivation to study polarized exclusive $\rho$ production is that this channel plays a rather prominent role in semi-inclusive pion production $[11,12,9]$, which has become a privileged tool to study a variety of spin effects, see e.g. [13]. It is important to identify 
kinematical regions where the exclusive channel $e p \rightarrow e p \rho \rightarrow e p \pi^{+} \pi^{-}$dominates semiinclusive observables, because in these regions great care must be taken when interpreting the data in terms of semi-inclusive factorization.

Even with an unpolarized target, the spin structure of the process $e p \rightarrow e p \rho \rightarrow e p \pi^{+} \pi^{-}$ is very rich, because the angular distribution of the final state contains information on the helicities of the exchanged virtual photon and of the $\rho$ meson, as was worked out in the classical analysis of Schilling and Wolf [14]. Yet more detailed information is available with target polarization [15]. Experiments on unpolarized targets have found that $s$-channel helicity is approximately conserved in the transition from the $\gamma^{*}$ to the $\rho$, with helicity changing amplitudes occurring at most at the $10 \%$ level $[16,17,18,19,20]$. This greatly simplifies the spin structure of the process. The aim of the present paper is to provide an analysis framework for exclusive $\rho$ production on a polarized nucleon target, making as explicit as possible the relation between the angular dependence of the cross section and the helicity amplitudes describing the hadronic subprocess $\gamma^{*} p \rightarrow \rho p$. We will present our results in a form that emphasizes the close similarity in structure between an unpolarized and a polarized target. Using the helicity basis for both virtual photon and meson, we also provide an alternative to the representation of the unpolarized cross section in [14].

The following section gives the definitions of the kinematics and polarization variables for the reaction under study. In Section 3 we define the helicity amplitudes and the spin density matrix elements describing the process and discuss some of their general properties. In Section 4 we express the angular distribution of the polarized cross section in terms of these spin density matrix elements and point out some salient features of this representation. The simplifications arising from distinguishing natural and unnatural parity exchange in the reaction are discussed in Section 5. A number of positivity bounds relating different spin density matrix elements are given in Section 6. In Section 7 we explain the complications arising from the distinction between target polarization relative to the momentum of either the incident lepton or the virtual photon. The role of non-resonant contributions in $\pi^{+} \pi^{-}$production is briefly discussed in Section 8. Our results are summarized in Section 9.

\section{Kinematics and target polarization}

Let us consider the electroproduction process

$$
e(l)+p(p) \rightarrow e\left(l^{\prime}\right)+p\left(p^{\prime}\right)+\rho\left(q^{\prime}\right)
$$

followed by the decay

$$
\rho\left(q^{\prime}\right) \rightarrow \pi^{+}(k)+\pi^{-}\left(k^{\prime}\right),
$$

where four-momenta are given in parentheses. Throughout this work we use the one-photon exchange approximation. All or results are equally valid for the production of a $\phi$ followed by the decay $\phi \rightarrow K^{+} K^{-}$. They also hold if the scattered proton is replaced by an inclusive system $X$ with four-momentum $p^{\prime}$, as explained at the end of Section 3.

To describe the kinematics we use the conventional variables for deep inelastic processes, $Q^{2}=-q^{2}, x_{B}=Q^{2} /(2 p \cdot q)$ and $y=(p \cdot q) /(p \cdot l)$. We neglect the lepton mass 


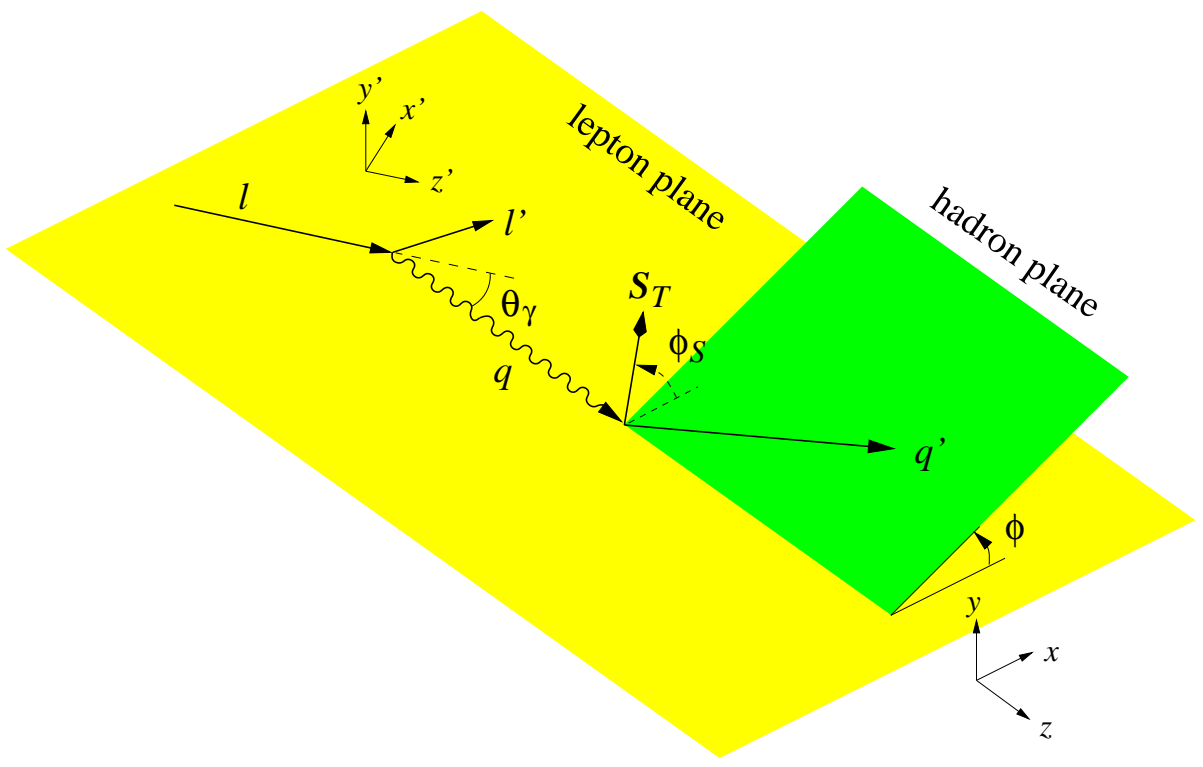

Figure 1: Kinematics of $e p \rightarrow e p \rho$ in the target rest frame. $\boldsymbol{S}_{T}$ is the transverse component of the target spin vector w.r.t. the virtual photon direction.

throughout and denote the longitudinal lepton beam polarization by $P_{\ell}$, with $P_{\ell}=+1$ corresponding to a purely right-handed and $P_{\ell}=-1$ to a purely left-handed beam. Let us now go to the target rest frame and introduce the right-handed coordinate system $(x, y, z)$ of Fig. 1 such that $\boldsymbol{q}$ points in the positive $z$ direction and $\boldsymbol{l}$ has a positive $x$ component. In this system we have $\boldsymbol{l}=|\boldsymbol{l}|\left(\sin \theta_{\gamma}, 0, \cos \theta_{\gamma}\right)$ and $\boldsymbol{q}=|\boldsymbol{q}|(0,0,1)$, where the angle $\theta_{\gamma}$ between $\boldsymbol{l}$ and $\boldsymbol{q}$ is defined to be between 0 and $\pi$. In accordance with the Trento convention [21] we define the angle $\phi$ between the lepton and the hadron plane as the azimuthal angle of $\boldsymbol{q}^{\prime}$ in this coordinate system, and $\phi_{S}$ as the azimuthal angle of the target spin vector $\boldsymbol{S}$. Following [22] we write $\boldsymbol{S}=\left(S_{T} \cos \phi_{S}, S_{T} \sin \phi_{S},-S_{L}\right)$ with $0 \leq S_{T} \leq 1$ and $-1 \leq S_{L} \leq 1$, so that $S_{T}$ and $S_{L}$ describe transverse and longitudinal polarization with respect to the virtual photon momentum, with $S_{L}=1$ corresponding to a right-handed proton in the $\gamma^{*} p$ c.m.
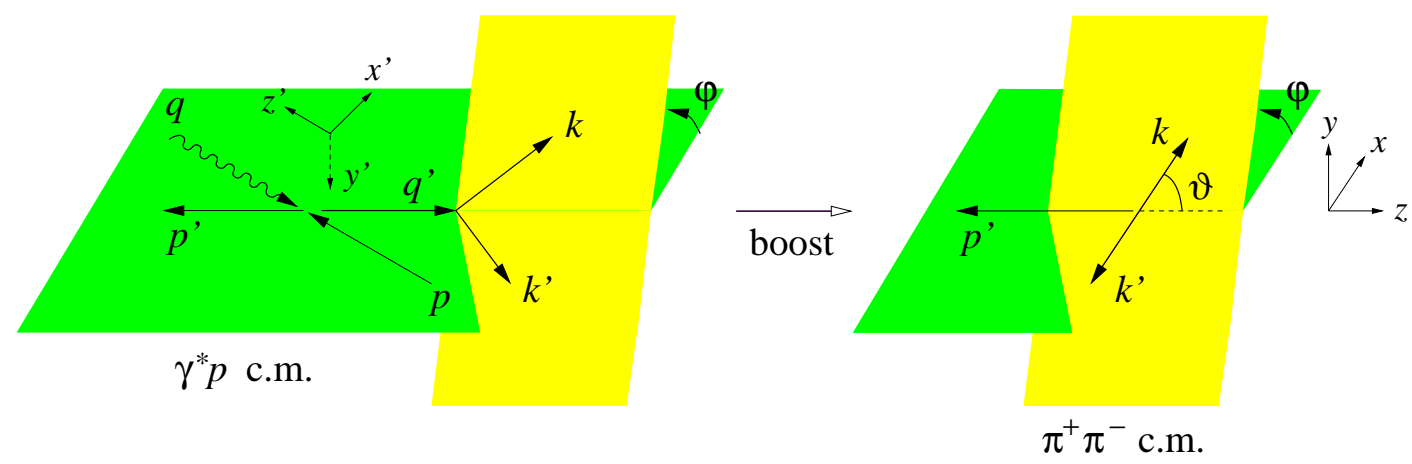

Figure 2: Kinematics of the hadronic subprocess $\gamma^{*} p \rightarrow \rho p$ followed by the decay $\rho \rightarrow \pi^{+} \pi^{-}$. The coordinate systems $(x, y, z)$ and $\left(x^{\prime}, y^{\prime}, z^{\prime}\right)$ differ from those in Fig. 1. 
To describe the target polarization of a given experimental setup, we introduce another right-handed coordinate system $\left(x^{\prime}, y^{\prime}, z^{\prime}\right)$ in the target rest frame such that $\boldsymbol{l}=$ $|\boldsymbol{l}|(0,0,1)$ and $\boldsymbol{q}=|\boldsymbol{q}|\left(-\sin \theta_{\gamma}, 0, \cos \theta_{\gamma}\right)$ as shown in Fig. 1. In this system we write $\boldsymbol{S}=\left(P_{T} \cos \psi, P_{T} \sin \psi,-P_{L}\right)$ with $0 \leq P_{T} \leq 1$ and $-1 \leq P_{L} \leq 1$, following again [22]. $P_{T}$ and $P_{L}$ describe transverse and longitudinal polarization with respect to the lepton beam direction, with $P_{L}=1$ corresponding to a right-handed proton in the ep c.m. The two sets of variables describing the target polarization are related by

$$
\begin{aligned}
S_{T} \cos \phi_{S} & =\cos \theta_{\gamma} P_{T} \cos \psi-\sin \theta_{\gamma} P_{L} \\
S_{T} \sin \phi_{S} & =P_{T} \sin \psi \\
S_{L} & =\sin \theta_{\gamma} P_{T} \cos \psi+\cos \theta_{\gamma} P_{L},
\end{aligned}
$$

which we will use in Sect. 7 . In terms of invariants the mixing angle $\theta_{\gamma}$ is given by

$$
\sin \theta_{\gamma}=\gamma \sqrt{\frac{1-y-\frac{1}{4} y^{2} \gamma^{2}}{1+\gamma^{2}}}, \quad \gamma=\frac{2 x_{B} M_{N}}{Q}
$$

where $M_{N}$ is the nucleon mass. In Bjorken kinematics $\gamma$ is small, and so is $\sin \theta_{\gamma} \approx \gamma \sqrt{1-y}$.

We finally specify the variables describing the vector meson decay (2.2). This is conveniently done in the $\pi^{+} \pi^{-}$c.m., which can be obtained from the $\gamma^{*} p$ c.m. by a boost in the direction of the scattered nucleon as shown in Fig. 2. In the $\pi^{+} \pi^{-}$c.m. we introduce the right-handed coordinate system $(x, y, z)$ shown in Fig. 2 , where $\boldsymbol{p}^{\prime}=\left|\boldsymbol{p}^{\prime}\right|(0,0,-1)$ and where the target momentum $\boldsymbol{p}$ has a positive $x$ component. In this system we define $\vartheta$ and $\varphi$ as the polar and azimuthal angle of the $\pi^{+}$momentum, i.e. $\boldsymbol{k}=|\boldsymbol{k}|(\sin \vartheta \cos \varphi, \sin \vartheta \sin \varphi, \cos \vartheta)$. The relation between our notation here and the one of Schilling and Wolf is ${ }^{1}$

$$
\phi_{\text {here }}=-\Phi_{[14]}, \quad \varphi_{\text {here }}=\phi_{[14]}, \quad \vartheta_{\text {here }}=\theta_{[14]} \cdot
$$

\section{Helicity amplitudes and spin density matrix}

The strong-interaction dynamics of the electroproduction process (2.1) is fully contained in the helicity amplitudes for the subprocess $\gamma^{*} p \rightarrow \rho p$. From these we will construct spin density matrix elements which describe the angular distribution of the overall reaction $e p \rightarrow e p \pi^{+} \pi^{-}$and its dependence on the target polarization.

Since we will deal with interference terms we must specify our phase conventions. We do this in the $\gamma^{*} p$ c.m. and use the right-handed coordinate system $\left(x^{\prime}, y^{\prime}, z^{\prime}\right)$ shown in Fig. 2. In this system we have $\boldsymbol{q}=|\boldsymbol{q}|(0,0,-1)$ and $\boldsymbol{q}^{\prime}=\left|\boldsymbol{q}^{\prime}\right|(\sin \Theta, 0,-\cos \Theta)$, with the scattering angle $\Theta$ of the vector meson defined to be between 0 and $\pi$. Note that the positive $z^{\prime}$ axis points along $\boldsymbol{p}$ rather than $\boldsymbol{q}$, as is often preferred for theoretical calculations. We specify polarization states of the target proton by two-component spinors $\chi_{+1 / 2}=(1,0)$

\footnotetext{
${ }^{1}$ We remark that the expression for $\sin \Phi$ given in eq. (13) of [14] is incorrect since it is always positive. A correct definition is given in [23].
} 
for positive and $\chi_{-1 / 2}=(0,1)$ for negative helicity. For the polarization vectors of the virtual photon we choose

$$
\begin{aligned}
\varepsilon_{+1} & =-\frac{1}{\sqrt{2}}(0,1,-i, 0), & & \varepsilon_{-1}=\frac{1}{\sqrt{2}}(0,1, i, 0), \\
\varepsilon_{0}^{\alpha} & =\mathcal{N}_{\varepsilon}\left(q^{\alpha}-\frac{q^{2}}{p \cdot q} p^{\alpha}\right), & &
\end{aligned}
$$

and for the polarization vectors of the $\rho$

$$
\begin{aligned}
e_{+1} & =-\frac{1}{\sqrt{2}}(0, \cos \Theta,-i, \sin \Theta), \quad e_{-1}=\frac{1}{\sqrt{2}}(0, \cos \Theta, i, \sin \Theta), \\
e_{0}^{\alpha} & =\mathcal{N}_{e}\left(q^{\prime \alpha}-\frac{q^{\prime 2}}{p^{\prime} \cdot q^{\prime}} p^{\prime \alpha}\right)
\end{aligned}
$$

where the subscripts indicate helicities. $\mathcal{N}_{\varepsilon}$ and $\mathcal{N}_{e}$ are positive constants ensuring the proper normalization $\varepsilon_{0}^{2}=1$ and $e_{0}^{2}=-1$ of the longitudinal polarization vectors. In the $\rho$ rest frame and the coordinate system $(x, y, z)$ of Fig. 2, our meson polarization vectors have the standard form $e_{+1}=-(0,1, i, 0) / \sqrt{2}, e_{-1}=(0,1,-i, 0) / \sqrt{2}$ and $e_{0}=(0,0,0,1)$. Our phase conventions for the proton and the virtual photon are as in [22].

We now introduce amplitudes $T_{\mu \lambda}^{\nu \sigma}$ for the subprocess $\gamma^{*}(\mu)+p(\lambda) \rightarrow \rho(\nu)+p(\sigma)$ with definite helicities $\mu, \nu, \lambda, \sigma$. Since the above phase conventions are defined with reference only to momentum vectors of this subprocess, the helicity amplitudes only depend on the photon virtuality, the $\gamma^{*} p$ scattering energy and the scattering angle $\Theta$, or equivalently on $Q^{2}, x_{B}$ and $t=\left(p-p^{\prime}\right)^{2}$. With our phase conventions they obey the usual parity relations

$$
T_{-\mu-\lambda}^{-\nu-\sigma}=(-1)^{\nu-\mu-\sigma+\lambda} T_{\mu \lambda}^{\nu \sigma}
$$

for equal $Q^{2}, x_{B}$ and $t$ on both sides. With these helicity amplitudes we define

$$
\rho_{\mu \mu^{\prime}, \lambda \lambda^{\prime}}^{\nu \nu^{\prime}}=\left(N_{T}+\epsilon N_{L}\right)^{-1} \sum_{\sigma} T_{\mu \lambda}^{\nu \sigma}\left(T_{\mu^{\prime} \lambda^{\prime}}^{\nu^{\prime} \sigma}\right)^{*}
$$

Regarding the upper indices this is the spin density matrix of the vector meson, whereas the lower indices specify the polarizations in the $\gamma^{*} p$ state from which the meson is produced. ${ }^{2}$ The normalization factors

$$
N_{T}=\frac{1}{2} \sum_{\lambda, \nu, \sigma}\left|T_{+\lambda}^{\nu \sigma}\right|^{2}, \quad N_{L}=\frac{1}{2} \sum_{\lambda, \nu, \sigma}\left|T_{0 \lambda}^{\nu \sigma}\right|^{2}
$$

are proportional to the differential cross sections $d \sigma_{T} / d t$ and $d \sigma_{L} / d t$ for transverse and longitudinal photon polarization, respectively, and

$$
\epsilon=\frac{1-y-\frac{1}{4} y^{2} \gamma^{2}}{1-y+\frac{1}{2} y^{2}+\frac{1}{4} y^{2} \gamma^{2}}
$$

\footnotetext{
${ }^{2}$ Taking the trace in the meson polarization indices we obtain the relation $\sum_{\nu} \rho_{\mu \mu^{\prime}, \lambda \lambda^{\prime}}^{\nu \nu} \propto d \sigma_{\mu^{\prime} \mu}^{\lambda^{\prime} \lambda} / d t$ between the spin density matrix $\rho$ introduced here and the cross sections and interference terms used in [22]. Compared with [22] we take the opposite order of indices in $\rho$, so that $\nu$ and $\nu^{\prime}$ appear in the standard order for a spin density matrix.
} 
is the usual ratio of longitudinal and transverse photon flux. In addition to $Q^{2}, x_{B}$ and $t$, the spin density matrix elements $\rho_{\mu \mu^{\prime}, \lambda \lambda^{\prime}}^{\nu \nu^{\prime}}$ depend on $\epsilon$ through the normalization factor $\left(N_{T}+\epsilon N_{L}\right)$. If one can perform a Rosenbluth separation by measuring at different $\epsilon$ but equal $Q^{2}$ and $x_{B}$, it is advantageous to normalize them instead to $N_{T}, N_{L}$ or $\sqrt{N_{T} N_{L}}$ as was done in [14]. It is straightforward to implement such a change in the formulae we give in the following.

We find it useful to introduce the combinations

$$
u_{\mu \mu^{\prime}}^{\nu \nu^{\prime}}=\frac{1}{2}\left(\rho_{\mu \mu^{\prime},++}^{\nu \nu^{\prime}}+\rho_{\mu \mu^{\prime},--}^{\nu \nu^{\prime}}\right), \quad \quad l_{\mu \mu^{\prime}}^{\nu \nu^{\prime}}=\frac{1}{2}\left(\rho_{\mu \mu^{\prime},++}^{\nu \nu^{\prime}}-\rho_{\mu \mu^{\prime},--}^{\nu \nu^{\prime}}\right)
$$

for an unpolarized and a longitudinally polarized target, where for the sake legibility we have labeled the target polarization by \pm instead of $\pm \frac{1}{2}$. The combinations

$$
s_{\mu \mu^{\prime}}^{\nu \nu^{\prime}}=\frac{1}{2}\left(\rho_{\mu \mu^{\prime},+-}^{\nu \nu^{\prime}}+\rho_{\mu \mu^{\prime},-+}^{\nu \nu^{\prime}}\right), \quad n_{\mu \mu^{\prime}}^{\nu \nu^{\prime}}=\frac{1}{2}\left(\rho_{\mu \mu^{\prime},+-}^{\nu \nu^{\prime}}-\rho_{\mu \mu^{\prime},-+}^{\nu \nu^{\prime}}\right)
$$

respectively describe transverse target polarization in the hadron plane ("sideways") and perpendicular to it ("normal"). One readily finds that the matrices $u, l$ and $s$ are hermitian, whereas $n$ is antihermitian,

$$
\begin{aligned}
& u_{\mu^{\prime} \mu}^{\nu^{\prime} \nu}=\left(u_{\mu \mu^{\prime}}^{\nu \nu^{\prime}}\right)^{*}, \quad l_{\mu^{\prime} \mu}^{\nu^{\prime} \nu}=\left(l_{\mu \mu^{\prime}}^{\nu \nu^{\prime}}\right)^{*}, \quad s_{\mu^{\prime} \mu}^{\nu^{\prime} \nu}=\left(s_{\mu \mu^{\prime}}^{\nu \nu^{\prime}}\right)^{*} \text {, } \\
& n_{\mu^{\prime} \mu}^{\nu^{\prime} \nu}=-\left(n_{\mu \mu^{\prime}}^{\nu \nu^{\prime}}\right)^{*} \text {. }
\end{aligned}
$$

The diagonal elements $u_{\mu \mu}^{\nu \nu}, l_{\mu \mu}^{\nu \nu}$ and $s_{\mu \mu}^{\nu \nu}$ are therefore purely real, whereas $n_{\mu \mu}^{\nu \nu}$ is purely imaginary. Furthermore, the parity relations (3.3) translate into

$$
\begin{aligned}
& u_{-\mu-\mu^{\prime}}^{-\nu-\nu^{\prime}}=(-1)^{\nu-\mu-\nu^{\prime}+\mu^{\prime}} u_{\mu \mu^{\prime}}^{\nu \nu^{\prime}}, \quad l_{-\mu-\mu^{\prime}}^{-\nu-\nu^{\prime}}=-(-1)^{\nu-\mu-\nu^{\prime}+\mu^{\prime}} l_{\mu \mu^{\prime}}^{\nu \nu^{\prime}}, \\
& n_{-\mu-\mu^{\prime}}^{-\nu-\nu^{\prime}}=(-1)^{\nu-\mu-\nu^{\prime}+\mu^{\prime}} n_{\mu \mu^{\prime}}^{\nu \nu^{\prime}}, \quad s_{-\mu-\mu^{\prime}}^{-\nu-\nu^{\prime}}=-(-1)^{\nu-\mu-\nu^{\prime}+\mu^{\prime}} s_{\mu \mu^{\prime}}^{\nu \nu^{\prime}} \text {. }
\end{aligned}
$$

As a consequence the matrix elements

$$
u_{-+}^{-+}, u_{-+}^{+-}, u_{00}^{-+}, u_{-+}^{00}
$$

are purely real, whereas the corresponding elements of $l, s$ and $n$ are purely imaginary.

Both experiment and theory indicate that $s$-channel helicity is approximately conserved in the $\gamma^{*} \rightarrow \rho$ transition for small invariant momentum transfer $t$. Correspondingly, one expects that spin density matrix elements involving the product of two helicity conserving amplitudes are greater than interference terms between a helicity conserving and a helicity changing amplitude, and that those are greater than matrix elements involving the product of two helicity changing amplitudes (where we refer to the helicities of the photon and the $\rho$ but not of the nucleon). Exceptions to this rule are however possible, since two large amplitudes can have a small interference term because of their relative phase, and since there can be cancellation of individually large terms in the linear combinations (3.7) and (3.8) associated with different target polarizations. With this caveat in mind one can readily assess the expected size of the spin density matrix elements (3.7) and (3.8) by comparing the upper with the lower indices. 
Let us now investigate the behavior of our matrix elements for $\Theta \rightarrow 0$, i.e. in the limit of forward scattering $\gamma^{*} p \rightarrow \rho p$. To this end we perform a partial wave decomposition

$$
T_{\mu \lambda}^{\nu \sigma}(\Theta)=\sum_{J} t_{\mu \lambda}^{\nu \sigma}(J) d_{\lambda-\mu, \sigma-\nu}^{J}(\Theta)
$$

where we have suppressed the dependence of $T$ and the partial wave amplitudes $t(J)$ on $Q^{2}$ and $x_{B}$. Using the behavior $d_{m, n}^{J}(\Theta) \sim \Theta^{|m-n|}$ of the rotation functions for $\Theta \rightarrow 0$ we readily find

$$
u_{\mu \mu^{\prime}}^{\nu \nu^{\prime}}, l_{\mu \mu^{\prime}}^{\nu \nu^{\prime}} \sim \Theta^{p}, \quad n_{\mu \mu^{\prime}}^{\nu \nu^{\prime}}, s_{\mu \mu^{\prime}}^{\nu \nu^{\prime}} \sim \Theta^{q}
$$

with

$$
\begin{aligned}
& p \geq p_{\min }=\min _{\sigma, \lambda= \pm 1 / 2}\left\{|\nu-\mu-\sigma+\lambda|+\left|\nu^{\prime}-\mu^{\prime}-\sigma+\lambda\right|\right\}, \\
& q \geq q_{\min }=\min _{\sigma, \lambda= \pm 1 / 2}\left\{|\nu-\mu-\sigma+\lambda|+\left|\nu^{\prime}-\mu^{\prime}-\sigma-\lambda\right|\right\} .
\end{aligned}
$$

With $\Theta \propto\left(t_{0}-t\right)^{1 / 2}$ for small $\Theta$, we can rewrite (3.13) as

$$
u_{\mu \mu^{\prime}}^{\nu \nu^{\prime}}, l_{\mu \mu^{\prime}}^{\nu \nu^{\prime}} \underset{t \rightarrow t_{0}}{\sim}\left(t_{0}-t\right)^{p / 2}, \quad n_{\mu \mu^{\prime}}^{\nu \nu^{\prime}}, s_{\mu \mu^{\prime}}^{\nu \nu^{\prime}} \underset{t \rightarrow t_{0}}{\sim}\left(t_{0}-t\right)^{q / 2},
$$

where $t_{0}$ is the value of $t$ for $\Theta=0$ at given $Q^{2}$ and $x_{B}$. In Tables 1 and 2 we give the corresponding powers for the linear combinations of spin density matrix elements that will appear in our results for the cross section in Section 4. We have ordered the entries according to the hierarchy discussed after (3.11), listing first terms containing the product of two helicity conserving amplitudes, then terms containing the interference between a helicity conserving and a helicity changing amplitude, and finally terms which only involve helicity changing amplitudes (with helicities always referring to the photon and the $\rho$ but not to the nucleon).

We emphasize that certain partial wave amplitudes $t_{\mu \lambda}^{\nu \sigma}(J)$ in (3.12) may be zero or negligibly small for dynamical reasons. The actual powers of $\left(t_{0}-t\right)^{1 / 2}$ in $(3.15)$ can thus be larger than the minimum values $p_{\text {min }}$ and $q_{\text {min }}$ required by angular momentum conservation. If there is for instance no $s$-channel helicity transferred between the protonproton and the photon-meson transitions, then the relevant powers for $n$ and $s$ are given by $q=p_{\min }+1$, which is equal to $q_{\min }+2$ for all but the first four entries in Tables 1 and 2 . A concrete realization of this scenario is the calculation in [24], where the proton-proton transition is described by the generalized parton distributions $H, E$ and $\tilde{H}, \tilde{E}$, which do not allow for helicity transfer to the photon-meson transition.

In the limit of large $Q^{2}$ at fixed $x_{B}$ and $t$, the proof of the factorization theorem in [2] implies that the transition from a longitudinal photon to a longitudinal $\rho$ becomes dominant, with all other transitions suppressed by powers of $1 / Q$. In this limit only the spin density matrix elements $u_{0}^{0} 0 \begin{aligned} & 0 \\ & 0\end{aligned}$ and $n_{0}^{0} 00$ survive and can be expressed as convolutions of hard-scattering kernels with generalized parton distributions and the light-cone distribution amplitude of the $\rho$. To leading order in $1 / Q$ one has in particular

$$
\frac{\operatorname{Im} n_{00}^{00}}{u_{00}^{00}}=\frac{\sqrt{t_{0}-t}}{M_{N}} \frac{\sqrt{1-\xi^{2}} \operatorname{Im}\left(\mathcal{E}^{*} \mathcal{H}\right)}{\left(1-\xi^{2}\right)|\mathcal{H}|^{2}-\left(\xi^{2}+t /\left(4 M_{N}^{2}\right)\right)|\mathcal{E}|^{2}-2 \xi^{2} \operatorname{Re}\left(\mathcal{E}^{*} \mathcal{H}\right)},
$$




\begin{tabular}{llc}
\hline \hline & \multicolumn{1}{c}{ matrix elements } & $p_{\min }$ \\
\hline$u_{++}^{00}+\epsilon u_{00}^{00}$ & $l_{0+}^{0+}-l_{0+}^{-0}$ & 0 \\
$u_{0+}^{0+}-u_{0+}^{-0}$ & 0 \\
$u_{++}^{++}+u_{++}^{--}+2 \epsilon u_{00}^{++}$ & $l_{++}^{++}+l_{++}^{--}$ & 0 \\
$u_{-+}^{-+}$ & $l_{-+}^{-+}$ & 0 \\
$u_{0+}^{00}$ & $l_{0+}^{00}$ & 1 \\
$u_{++}^{0+}-u_{++}^{-0}+2 \operatorname{Re} \epsilon u_{0+}^{0+}$ & $l_{++}^{0+}-l_{++}^{-0}+2 i \operatorname{Im} \epsilon l_{00}^{0+}$ & 1 \\
$u_{-+}^{0+}$ & $l_{-+}^{0+}$ & 1 \\
$u_{0+}^{0-}-u_{0+}^{+0}$ & $l_{0+}^{0-}-l_{0+}^{+0}$ & 2 \\
$u_{++}^{-+}+\epsilon u_{00}^{-+}$ & $l_{++}^{-+}+\epsilon l_{00}^{-+}$ & 2 \\
$u_{-+}^{++}$ & $l_{-+}^{++}$ & 2 \\
$u_{0+}^{++}+u_{0+}^{--}$ & $l_{0+}^{++}+l_{0+}^{--}$ & 1 \\
$u_{0+}^{-+}$ & $l_{0+}^{-+}$ & 1 \\
& $l_{++}^{00}$ & 2 \\
$u_{-+}^{00}$ & $l_{-+}^{00}$ & 2 \\
$u_{-+}^{+0}$ & $l_{-+}^{+0}$ & 3 \\
$u_{0+}^{++}$ & $l_{0+}^{+-}$ & 3 \\
$u_{-+}^{+-}$ & $l_{-+}^{+-}$ & 4 \\
\hline \hline
\end{tabular}

Table 1: Minimum values of the powers which control the $t \rightarrow t_{0}$ behavior of combinations of spin density matrix elements $u$ and $l$ as in (3.15). Some of the combinations are purely real or purely imaginary because of the symmetry relations (3.9) and (3.10), whereas others are complex valued.

where $\xi=x_{B} /\left(2-x_{B}\right)$ and the convolution integrals $\mathcal{H}$ and $\mathcal{E}$ are for instance given in [22]. Experimental results and phenomenological analysis show however that $1 / Q^{2}$ suppressed effects can be numerically significant for $Q^{2}$ of several $\mathrm{GeV}^{2}$, see e.g. [25, 24, 9, 10]. This concerns both power corrections within $u_{00}^{00}$ or $n_{00}^{00}$ and formally power suppressed spin density matrix elements such as $u_{++}^{++}$or $u_{0+}^{0+}$. The detailed analysis in [2] reveals that beyond leading-power accuracy in $1 / Q$, factorization of meson production into a hardscattering subprocess and nonperturbative quantities pertaining either to the target or to the meson may be broken. On the other hand, factorization based approaches which go beyond leading power in $1 / Q$ and in particular also evaluate transition amplitudes for transverse polarization of the $\gamma^{*}$ or $\rho$ have been phenomenologically rather successful, see e.g. $[26,24]$

Let us finally generalize our considerations to the process

$$
e(l)+p(p) \rightarrow e\left(l^{\prime}\right)+X\left(p^{\prime}\right)+\rho\left(q^{\prime}\right)
$$

where the target proton dissociates into a hadronic system $X$. In analogy to the elastic case one can introduce helicity amplitudes $T_{\mu \lambda}^{\nu \sigma, X}$ and combine them into spin density matrix 


\begin{tabular}{|c|c|c|}
\hline \multicolumn{2}{|c|}{ matrix elements } & \multirow{2}{*}{$\frac{q_{\min }}{1}$} \\
\hline $\begin{array}{ll}n_{++}^{00}+\epsilon n_{00}^{00} & 0\end{array}$ & & \\
\hline$n_{0+}^{0+}-n_{0+}^{-0}$ & $s_{0+}^{0+}-s_{0+}^{-0}$ & 1 \\
\hline$n_{++}^{++}+n_{++}^{--}+2 \epsilon n_{00}^{++}$ & $s_{++}^{++}+s_{++}^{--}$ & 1 \\
\hline$n_{-+}^{-+}$ & $s_{-+}^{-+}$ & 1 \\
\hline$n_{0+}^{00}$ & $s_{0+}^{00}$ & 0 \\
\hline$n_{++}^{0+}-n_{++}^{-0}+2 i \operatorname{Im} \epsilon n_{00}^{0+}$ & $s_{++}^{0+}-s_{++}^{-0}+2 i \operatorname{Im} \epsilon s_{00}^{0+}$ & 0 \\
\hline$n_{-+}^{0+}$ & $s_{-+}^{0+}$ & 0 \\
\hline$n_{0+}^{0-}-n_{0+}^{+0}$ & $s_{0+}^{0-}-s_{0+}^{+0}$ & 1 \\
\hline$n_{++}^{-+}+\epsilon n_{00}^{-+}$ & $s_{++}^{-+}+\epsilon s_{00}^{-+}$ & 1 \\
\hline$n_{-+}^{++}$ & $s_{-+}^{++}$ & 1 \\
\hline$n_{0+}^{++}+n_{0+}^{--}$ & $s_{0+}^{++}+s_{0+}^{--}$ & 0 \\
\hline \multirow[t]{2}{*}{$n_{0+}^{-+}$} & $s_{0+}^{-+}$ & 0 \\
\hline & $s_{++}^{00}$ & 1 \\
\hline$n_{-+}^{00}$ & $s_{-+}^{00}$ & 1 \\
\hline$n_{-+}^{+0}$ & $s_{-+}^{+0}$ & 2 \\
\hline$n_{0+}^{+-}$ & $s_{0+}^{+-}$ & 2 \\
\hline$n_{-+}^{+-}$ & $s_{-+}^{+-}$ & 3 \\
\hline
\end{tabular}

Table 2: As Table 1 but for combinations of spin density matrix elements $n$ and $s$.

elements

$$
\rho_{\mu \mu^{\prime}, \lambda \lambda^{\prime}}^{\nu \nu^{\prime}}=\left(N_{T}+\epsilon N_{L}\right)^{-1} \sum_{X, \sigma} T_{\mu \lambda}^{\nu \sigma, X}\left(T_{\mu^{\prime} \lambda^{\prime}}^{\nu^{\prime} \sigma}\right)^{*} .
$$

The normalization factors $N_{T}$ and $N_{L}$ are defined as in (3.5) but with an additional sum over all hadronic states $X$ of given invariant mass $M_{X}$, on which $\rho_{\mu \mu^{\prime}, \lambda \lambda^{\prime}}^{\nu \nu^{\prime}}$ now depends in addition to $Q^{2}, x_{B}, t$ and $\epsilon$. The combinations (3.7) and (3.8) for different target polarization have the same symmetry properties (3.9) and (3.10) as in the elastic case. Their behavior for $t \rightarrow t_{0}$ can be different, since in (3.14) one must now take the minimum over all possible helicities $\sigma= \pm \frac{1}{2}, \pm \frac{3}{2}, \ldots$ of the hadronic system $X$. One finds however that the powers $p_{\min }$ and $q_{\min }$ for the combinations of spin density matrix elements in Tables 1 and 2 are the same as in the elastic case. The results in the remainder of this work only depend on the properties (3.9) and (3.10) and thus immediately generalize to the case of target dissociation.

\section{The angular distribution}

The calculation of the cross section for $e p \rightarrow e p \pi^{+} \pi^{-}$proceeds by using standard methods and we shall only sketch the essential steps. More details are for instance given in $[14,27$, 
22]. With our phase conventions the polarization state of the proton target is described by the spin density matrix

$$
\tau_{\lambda \lambda^{\prime}}=\frac{1}{2}\left(\begin{array}{cc}
1+S_{L} & S_{T} e^{-i\left(\phi-\phi_{S}\right)} \\
S_{T} e^{i\left(\phi-\phi_{S}\right)} & 1-S_{L}
\end{array}\right),
$$

which is to be contracted with the matrix in (3.4). The result is conveniently expressed in terms of the combinations (3.7) and (3.8) as

$$
\sum_{\lambda, \lambda^{\prime}} \tau_{\lambda \lambda^{\prime}} \rho_{\mu \mu^{\prime}, \lambda \lambda^{\prime}}^{\nu \nu^{\prime}}=u_{\mu \mu^{\prime}}^{\nu \nu^{\prime}}+S_{L} l_{\mu \mu^{\prime}}^{\nu \nu^{\prime}}+S_{T} \cos \left(\phi-\phi_{S}\right) s_{\mu \mu^{\prime}}^{\nu \nu^{\prime}}-S_{T} \sin \left(\phi-\phi_{S}\right) i n_{\mu \mu^{\prime}}^{\nu \nu^{\prime}}
$$

and describes the subprocess $\gamma^{*} p \rightarrow \rho p$. The decay $\rho \rightarrow \pi^{+} \pi^{-}$is taken into account by multiplication with the spherical harmonics,

$$
\rho_{\mu \mu^{\prime}}=\sum_{\nu, \nu^{\prime}} \sum_{\lambda, \lambda^{\prime}} \tau_{\lambda \lambda^{\prime}} \rho_{\mu \mu^{\prime}, \lambda \lambda^{\prime}}^{\nu \nu^{\prime}} Y_{1 \nu}(\varphi, \vartheta) Y_{1 \nu^{\prime}}^{*}(\varphi, \vartheta)
$$

where

$$
Y_{1+1}=-\sqrt{\frac{3}{8 \pi}} \sin \vartheta e^{i \varphi}, \quad Y_{10}=\sqrt{\frac{3}{4 \pi}} \cos \vartheta, \quad Y_{1-1}=\sqrt{\frac{3}{8 \pi}} \sin \vartheta e^{-i \varphi} .
$$

To obtain the cross section for the overall process $e p \rightarrow e p \pi^{+} \pi^{-}$one must finally contract the matrix $\rho_{\mu \mu^{\prime}}$ in $(4.3)$ with the spin density matrix of the virtual photon. ${ }^{3}$ The cross section can be written as

$$
\begin{aligned}
& \frac{d \sigma}{d \psi d \phi d \varphi d(\cos \vartheta) d x_{B} d Q^{2} d t}=\frac{1}{(2 \pi)^{2}} \frac{d \sigma}{d x_{B} d Q^{2} d t} \\
& \times\left(W_{U U}+P_{\ell} W_{L U}+S_{L} W_{U L}+P_{\ell} S_{L} W_{L L}+S_{T} W_{U T}+P_{\ell} S_{T} W_{L T}\right)
\end{aligned}
$$

with

$$
\frac{d \sigma}{d x_{B} d Q^{2} d t}=\frac{\alpha_{\mathrm{em}}}{2 \pi} \frac{y^{2}}{1-\epsilon} \frac{1-x_{B}}{x_{B}} \frac{1}{Q^{2}}\left(\frac{d \sigma_{T}}{d t}+\epsilon \frac{d \sigma_{L}}{d t}\right),
$$

where $d \sigma_{T} / d t$ and $d \sigma_{L} / d t$ are the usual $\gamma^{*} p$ cross sections for a transverse and longitudinal photon and an unpolarized proton, with Hand's convention for virtual photon flux. The angular distribution is described by the quantities $W_{X Y}$, where $X$ specifies the beam and $Y$ the target polarization. The normalization of the unpolarized term $W_{U U}$ is

$$
\int \frac{d \phi}{2 \pi} \int d \varphi d(\cos \vartheta) W_{U U}(\phi, \varphi, \vartheta)=1
$$

To limit the length of subsequent expressions, we further decompose the coefficients according to the $\rho$ polarization and write

$$
\begin{aligned}
& W_{X Y}(\phi, \varphi, \vartheta) \\
& =\frac{3}{4 \pi}\left[\cos ^{2} \vartheta W_{X Y}^{L L}(\phi)+\sqrt{2} \cos \vartheta \sin \vartheta W_{X Y}^{L T}(\phi, \varphi)+\sin ^{2} \vartheta W_{X Y}^{T T}(\phi, \varphi)\right]
\end{aligned}
$$

for $X, Y=U, L$. The production of a longitudinal $\rho$ is described by $W_{X Y}^{L L}$, the production

\footnotetext{
${ }^{3} \mathrm{Up}$ to a global factor, the result of this contraction can e.g. be obtained from eq. (3.20) of [27], with $\rho_{\mu \mu^{\prime}}$ in the present work corresponding to $\sigma_{\mu^{\prime} \mu}^{(X)}$ in [27] and $\phi_{\text {here }}=-\varphi_{[27]}$.
} 
of a transverse $\rho$ (including the interference between positive and negative $\rho$ helicity) by $W_{X Y}^{T T}$, and the interference between longitudinal and transverse $\rho$ polarization by $W_{X Y}^{L T}$. For a transversely polarized target we have in addition a dependence on $\phi_{S}$,

$$
\begin{aligned}
& W_{X T}\left(\phi_{S}, \phi, \varphi, \vartheta\right) \\
& =\frac{3}{4 \pi}\left[\cos ^{2} \vartheta W_{X T}^{L L}\left(\phi_{S}, \phi\right)+\sqrt{2} \cos \vartheta \sin \vartheta W_{X T}^{L T}\left(\phi_{S}, \phi, \varphi\right)+\sin ^{2} \vartheta W_{X T}^{T T}\left(\phi_{S}, \phi, \varphi\right)\right]
\end{aligned}
$$

with $X=U, L$. In addition to the angles, all coefficients $W_{X Y}$ depend on $Q^{2}, x_{B}$ and $t$, which we have not displayed for the sake of legibility.

For unpolarized target and beam we have

$$
\begin{aligned}
& W_{U U}^{L L}(\phi)=\left(u_{++}^{00}+\epsilon u_{00}^{0} 0\right)-2 \cos \phi \sqrt{\epsilon(1+\epsilon)} \operatorname{Re} u_{0+}^{00}-\cos (2 \phi) \epsilon u_{-+}^{00}, \\
& W_{U U}^{L T}(\phi, \varphi)=\cos (\phi+\varphi) \sqrt{\epsilon(1+\epsilon)} \operatorname{Re}\left(u_{0+}^{0+}-u_{0+}^{-0}\right) \\
& -\cos \varphi \operatorname{Re}\left(u_{++}^{0+}-u_{++}^{-0}+2 \epsilon u_{00}^{0+}\right)+\cos (2 \phi+\varphi) \epsilon \operatorname{Re} u_{-+}^{0+} \\
& -\cos (\phi-\varphi) \sqrt{\epsilon(1+\epsilon)} \operatorname{Re}\left(u_{0+}^{0-}-u_{0+}^{+0}\right)+\cos (2 \phi-\varphi) \epsilon \operatorname{Re} u_{-+}^{+0}, \\
& W_{U U}^{T T}(\phi, \varphi)=\frac{1}{2}\left(u_{++}^{++}+u_{++}^{--}+2 \epsilon u_{00}^{++}\right)+\frac{1}{2} \cos (2 \phi+2 \varphi) \epsilon u_{-+}^{-+} \\
& -\cos \phi \sqrt{\epsilon(1+\epsilon)} \operatorname{Re}\left(u_{0+}^{++}+u_{0+}^{--}\right)+\cos (\phi+2 \varphi) \sqrt{\epsilon(1+\epsilon)} \operatorname{Re} u_{0+}^{-+} \\
& -\cos (2 \varphi) \operatorname{Re}\left(u_{++}^{-+}+\epsilon u_{00}^{-+}\right)-\cos (2 \phi) \epsilon \operatorname{Re} u_{-+}^{++} \\
& +\cos (\phi-2 \varphi) \sqrt{\epsilon(1+\epsilon)} \operatorname{Re} u_{0+}^{+-}+\frac{1}{2} \cos (2 \phi-2 \varphi) \epsilon u_{-+}^{+-} .
\end{aligned}
$$

Here and in the following we order terms according to the hierarchy discussed after (3.11), as already done in Table 1 . The terms independent of $\phi$ and $\varphi$ in $W_{U U}^{L L}$ and $W_{U U}^{T T}$ are related by

$$
u_{++}^{++}+u_{++}^{--}+2 \epsilon u_{00}^{++}=1-\left(u_{++}^{0} 0 \quad+\epsilon u_{0}^{0} 00\right),
$$

which ensures the normalization condition (4.7). The terms for beam polarization with an unpolarized target read

$$
\begin{aligned}
W_{L U}^{L L}(\phi)= & -2 \sin \phi \sqrt{\epsilon(1-\epsilon)} \operatorname{Im} u_{0+}^{00} \\
W_{L U}^{L T}(\phi, \varphi)= & \sin (\phi+\varphi) \sqrt{\epsilon(1-\epsilon)} \operatorname{Im}\left(u_{0+}^{0+}-u_{0+}^{-0}\right) \\
& -\sin \varphi \sqrt{1-\epsilon^{2}} \operatorname{Im}\left(u_{++}^{0+}-u_{++}^{-0}\right) \\
& -\sin (\phi-\varphi) \sqrt{\epsilon(1-\epsilon)} \operatorname{Im}\left(u_{0+}^{0-}-u_{0+}^{+0}\right) \\
W_{L U}^{T T}(\phi, \varphi)= & -\sin \phi \sqrt{\epsilon(1-\epsilon)} \operatorname{Im}\left(u_{0+}^{++}+u_{0+}^{--}\right)+\sin (\phi+2 \varphi) \sqrt{\epsilon(1-\epsilon)} \operatorname{Im} u_{0+}^{-+} \\
& -\sin (2 \varphi) \sqrt{1-\epsilon^{2}} \operatorname{Im} u_{++}^{-+} \\
& +\sin (\phi-2 \varphi) \sqrt{\epsilon(1-\epsilon)} \operatorname{Im} u_{0+}^{+-} .
\end{aligned}
$$


The results for longitudinal target polarization are very similar, with

$$
\begin{aligned}
W_{U L}^{L L}(\phi)= & -2 \sin \phi \sqrt{\epsilon(1+\epsilon)} \operatorname{Im} l_{0+}^{00}-\sin (2 \phi) \epsilon \operatorname{Im} l_{-+}^{00}, \\
W_{U L}^{L T}(\phi, \varphi)= & \sin (\phi+\varphi) \sqrt{\epsilon(1+\epsilon)} \operatorname{Im}\left(l_{0+}^{0+}-l_{0+}^{-0}\right) \\
& -\sin \varphi \operatorname{Im}\left(l_{++}^{0+}-l_{++}^{-0}+2 \epsilon l_{00}^{0+}\right)+\sin (2 \phi+\varphi) \epsilon \operatorname{Im} l_{-+}^{0+} \\
& -\sin (\phi-\varphi) \sqrt{\epsilon(1+\epsilon)} \operatorname{Im}\left(l_{0+}^{0-}-l_{0+}^{+0}\right)+\sin (2 \phi-\varphi) \epsilon \operatorname{Im} l_{-+}^{+0}, \\
W_{U L}^{T T}(\phi, \varphi)= & \frac{1}{2} \sin (2 \phi+2 \varphi) \epsilon \operatorname{Im} l_{-+}^{-+} \\
& -\sin \phi \sqrt{\epsilon(1+\epsilon)} \operatorname{Im}\left(l_{0+}^{++}+l_{0+}^{--}\right)+\sin (\phi+2 \varphi) \sqrt{\epsilon(1+\epsilon)} \operatorname{Im} l_{0+}^{-+} \\
& -\sin (2 \varphi) \operatorname{Im}\left(l_{++}^{-+}+\epsilon l_{00}^{-+}\right)-\sin (2 \phi) \epsilon \operatorname{Im} l_{-+}^{++} \\
& +\sin (\phi-2 \varphi) \sqrt{\epsilon(1+\epsilon)} \operatorname{Im} l_{0+}^{+-}+\frac{1}{2} \sin (2 \phi-2 \varphi) \epsilon \operatorname{Im} l_{-+}^{+-}
\end{aligned}
$$

for an unpolarized beam, and

$$
\begin{aligned}
W_{L L}^{L L}(\phi)= & -2 \cos \phi \sqrt{\epsilon(1-\epsilon)} \operatorname{Re} l_{0+}^{00}+\sqrt{1-\epsilon^{2}} l_{++}^{00}, \\
W_{L L}^{L T}(\phi, \varphi)= & \cos (\phi+\varphi) \sqrt{\epsilon(1-\epsilon)} \operatorname{Re}\left(l_{0+}^{0+}-l_{0+}^{-0}\right) \\
& -\cos \varphi \sqrt{1-\epsilon^{2}} \operatorname{Re}\left(l_{++}^{0+}-l_{++}^{-0}\right) \\
& -\cos (\phi-\varphi) \sqrt{\epsilon(1-\epsilon)} \operatorname{Re}\left(l_{0+}^{0-}-l_{0+}^{+0}\right) \\
W_{L L}^{T T}(\phi, \varphi)= & \sqrt{1-\epsilon^{2}} \frac{1}{2}\left(l_{++}^{++}+l_{++}^{--}\right) \\
& -\cos \phi \sqrt{\epsilon(1-\epsilon)} \operatorname{Re}\left(l_{0+}^{++}+l_{0+}^{--}\right)+\cos (\phi+2 \varphi) \sqrt{\epsilon(1-\epsilon)} \operatorname{Re} l_{0+}^{-+} \\
& -\cos (2 \varphi) \sqrt{1-\epsilon^{2}} \operatorname{Re} l_{++}^{-+} \\
& +\cos (\phi-2 \varphi) \sqrt{\epsilon(1-\epsilon)} \operatorname{Re} l_{0+}^{+-}
\end{aligned}
$$

for beam polarization. In (4.10) to (4.14) we have used the symmetry relations (3.9) and (3.10) to write our results with a minimal set of matrix elements $u_{\mu \mu^{\prime}}^{\nu \nu^{\prime}}$ or $l_{\mu \mu^{\prime}}^{\nu \nu^{\prime}}$. Although they are a little lengthy, their structure is quite simple:

1. The combinations $u_{\mu \mu^{\prime}}^{++}+u_{\mu \mu^{\prime}}^{--}, u_{\mu \mu^{\prime}}^{0+}-u_{\mu \mu^{\prime}}^{-0}$ and $u_{\mu \mu^{\prime}}^{0-}-u_{\mu \mu^{\prime}}^{+0}$ and their analogs for $l$ always appear together because the corresponding products of spherical harmonics are identical, $Y_{1+1} Y_{1+1}^{*}=Y_{1-1} Y_{1-1}^{*}$ and $Y_{10} Y_{1+1}^{*}=-Y_{1-1} Y_{10}^{*}$. In some cases the corresponding sum can be simplified using symmetry relations like $u_{00}^{++}+u_{00}^{--}=$ $2 u_{00}^{++}$, but in others one remains with a linear combination of matrix elements that cannot be separated. With the caveats discussed after (3.11) one finds however that these combinations are dominated by a single term. Exceptions are $\operatorname{Re}\left(u_{++}^{0+}-u_{++}^{-0}+\right.$ $\left.2 \epsilon u_{00}^{0+}\right)$ and $\operatorname{Im}\left(l_{++}^{0+}-l_{++}^{-0}+2 \epsilon l_{00}^{0+}\right)$, each of which contains two interference terms between a helicity conserving and a helicity changing amplitude. 
2. An angular dependence through $(k \phi+m \varphi)$ is associated with the interference between transverse and longitudinal $\rho$ polarization for $|m|=1$, the interference between positive and negative $\rho$ helicity for $|m|=2$, and equal $\rho$ polarization in the amplitude and its conjugate for $m=0$. In the same way $|k|=1,|k|=2$ and $k=0$ are related to the virtual photon polarization. Notice that for $m=0$ one can distinguish transverse and longitudinal $\rho$ production by the $\vartheta$ dependence in (4.8), whereas for $k=0$ the separation of terms for transverse and longitudinal photons requires variation of $\epsilon$. The beam spin asymmetries $W_{L U}$ and $W_{L L}$ contain no terms with $|k|=2$, because there is no term with $P_{\ell} \cos 2 \phi$ or $P_{\ell} \sin 2 \phi$ in the spin density matrix of the virtual photon.

3. The unpolarized or doubly polarized terms $W_{U U}$ and $W_{L L}$ depend on $\operatorname{Re} u$ or $\operatorname{Re} l$ and are even under the reflection $(\phi, \varphi) \rightarrow(-\phi,-\varphi)$ of the azimuthal angles, whereas the single spin asymmetries $W_{L U}$ and $W_{U L}$ depend on $\operatorname{Im} u$ or $\operatorname{Im} l$ and are odd under $(\phi, \varphi) \rightarrow(-\phi,-\varphi)$. This is a consequence of parity and time reversal invariance.

4. As we have written our results, the angular distribution for longitudinal target polarization can be obtained from the one for an unpolarized target by replacing

$$
\begin{aligned}
& \cos (k \phi+m \varphi) \operatorname{Re} u \rightarrow \sin (k \phi+m \varphi) \operatorname{Im} l, \\
& \sin (k \phi+m \varphi) \operatorname{Im} u \rightarrow \cos (k \phi+m \varphi) \operatorname{Re} l .
\end{aligned}
$$

Terms with $k=m=0$ in $W_{U U}$ and $W_{L L}$ are independent of $\phi$ and $\varphi$, and have of course no counterparts in $W_{U L}$ or $W_{L U}$. This corresponds to 16 terms with a different angular dependence in $W_{U U}$ and 14 terms in $W_{U L}$, and to 10 terms in $W_{L L}$ and 8 terms in $W_{L U}$.

The symmetry properties (3.9) and (3.10), which we used to obtain (4.10) to (4.14), are identical for $u_{\mu \mu^{\prime}}^{\nu \nu^{\prime}}$ and $i n_{\mu \mu^{\prime}}^{\nu \nu^{\prime}}$, as well as for $l_{\mu \mu^{\prime}}^{\nu \nu^{\prime}}$ and $s_{\mu \mu^{\prime}}^{\nu \nu^{\prime}}$. According to (4.2) the cross section for a transversely polarized target can therefore be obtained from the one for longitudinal and no target polarization by the replacements

$$
\begin{array}{ll}
\operatorname{Re} u \rightarrow S_{T} \sin \left(\phi-\phi_{S}\right) \operatorname{Im} n, & S_{L} \operatorname{Im} l \rightarrow S_{T} \cos \left(\phi-\phi_{S}\right) \operatorname{Im} s, \\
\operatorname{Im} u \rightarrow-S_{T} \sin \left(\phi-\phi_{S}\right) \operatorname{Re} n, & S_{L} \operatorname{Re} l \rightarrow S_{T} \cos \left(\phi-\phi_{S}\right) \operatorname{Re} s .
\end{array}
$$

We thus simply have

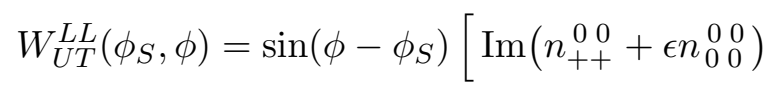

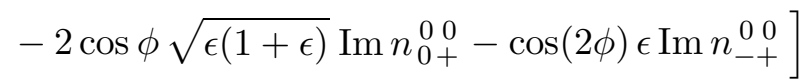

$$
\begin{aligned}
& +\cos \left(\phi-\phi_{S}\right)\left[-2 \sin \phi \sqrt{\epsilon(1+\epsilon)} \operatorname{Im} s_{0+}^{00}-\sin (2 \phi) \epsilon \operatorname{Im} s_{-+}^{00}\right], \\
& W_{U T}^{L T}\left(\phi_{S}, \phi, \varphi\right)=\sin \left(\phi-\phi_{S}\right)\left[\cos (\phi+\varphi) \sqrt{\epsilon(1+\epsilon)} \operatorname{Im}\left(n_{0+}^{0+}-n_{0+}^{-0}\right)\right. \\
& -\cos \varphi \operatorname{Im}\left(n_{++}^{0+}-n_{++}^{-0}+2 \epsilon n_{00}^{0+}\right)+\cos (2 \phi+\varphi) \epsilon \operatorname{Im} n_{-+}^{0+} \\
& \left.-\cos (\phi-\varphi) \sqrt{\epsilon(1+\epsilon)} \operatorname{Im}\left(n_{0+}^{0-}-n_{0+}^{+0}\right)+\cos (2 \phi-\varphi) \epsilon \operatorname{Im} n_{-+}^{+0}\right]
\end{aligned}
$$




$$
\begin{aligned}
&+ \cos \left(\phi-\phi_{S}\right)\left[\sin (\phi+\varphi) \sqrt{\epsilon(1+\epsilon)} \operatorname{Im}\left(s_{0+}^{0+}-s_{0+}^{-0}\right)\right. \\
&-\sin \varphi \operatorname{Im}\left(s_{++}^{0+}-s_{++}^{-0}+2 \epsilon s_{00}^{0+}\right)+\sin (2 \phi+\varphi) \epsilon \operatorname{Im} s_{-+}^{0+} \\
&\left.-\sin (\phi-\varphi) \sqrt{\epsilon(1+\epsilon)} \operatorname{Im}\left(s_{0+}^{0-}-s_{0+}^{+0}\right)+\sin (2 \phi-\varphi) \epsilon \operatorname{Im} s_{-+}^{+0}\right] \\
& W_{U T}^{T T}\left(\phi_{S}, \phi, \varphi\right)=\sin \left(\phi-\phi_{S}\right)\left[\frac{1}{2} \operatorname{Im}\left(n_{++}^{++}+n_{++}^{--}+2 \epsilon n_{00}^{++}\right)+\frac{1}{2} \cos (2 \phi+2 \varphi) \epsilon \operatorname{Im} n_{-+}^{-+}\right. \\
& \quad-\cos \phi \sqrt{\epsilon(1+\epsilon)} \operatorname{Im}\left(n_{0+}^{++}+n_{0+}^{--}\right)+\cos (\phi+2 \varphi) \sqrt{\epsilon(1+\epsilon)} \operatorname{Im} n_{0+}^{-+} \\
& \quad-\cos (2 \varphi) \operatorname{Im}\left(n_{++}^{-+}+\epsilon n_{00}^{-+}\right)-\cos (2 \phi) \epsilon \operatorname{Im} n_{-+}^{++} \\
&\left.\quad+\cos (\phi-2 \varphi) \sqrt{\epsilon(1+\epsilon)} \operatorname{Im} n_{0+}^{+-}+\frac{1}{2} \cos (2 \phi-2 \varphi) \epsilon \operatorname{Im} n_{-+}^{+-}\right] \\
&+\cos \left(\phi-\phi \phi_{S}\right)\left[\frac{1}{2} \sin (2 \phi+2 \varphi) \epsilon \operatorname{Im} s_{-+}^{-+}\right. \\
& \quad-\sin \phi \sqrt{\epsilon(1+\epsilon)} \operatorname{Im}\left(s_{0+}^{++}+s_{0+}^{--}\right)+\sin (\phi+2 \varphi) \sqrt{\epsilon(1+\epsilon)} \operatorname{Im} s_{0+}^{-+} \\
& \quad-\sin (2 \varphi) \operatorname{Im}\left(s_{++}^{-+}+\epsilon s_{00}^{-+}\right)-\sin (2 \phi) \epsilon \operatorname{Im} s_{-+}^{++} \\
&\left.+\sin (\phi-2 \varphi) \sqrt{\epsilon(1+\epsilon)} \operatorname{Im} s_{0+}^{+-}+\frac{1}{2} \sin (2 \phi-2 \varphi) \epsilon \operatorname{Im} s_{-+}^{+-}\right]
\end{aligned}
$$

for an unpolarized beam, and

$$
\begin{aligned}
& W_{L T}^{L L}\left(\phi_{S}, \phi\right)= \sin \left(\phi-\phi_{S}\right)\left[2 \sin \phi \sqrt{\epsilon(1-\epsilon)} \operatorname{Re} n_{0+}^{0} 0\right] \\
&+ \cos \left(\phi-\phi_{S}\right)\left[-2 \cos \phi \sqrt{\epsilon(1-\epsilon)} \operatorname{Re} s_{0+}^{0} 0+\sqrt{1-\epsilon^{2}} s_{++}^{00}\right] \\
& W_{L T}^{L T}\left(\phi_{S}, \phi, \varphi\right)=\sin \left(\phi-\phi_{S}\right)\left[-\sin (\phi+\varphi) \sqrt{\epsilon(1-\epsilon)} \operatorname{Re}\left(n_{0+}^{0+}-n_{0+}^{-0}\right)\right. \\
&+\sin \varphi \sqrt{1-\epsilon^{2}} \operatorname{Re}\left(n_{++}^{0+}-n_{++}^{-0}\right) \\
&\left.\quad+\sin (\phi-\varphi) \sqrt{\epsilon(1-\epsilon)} \operatorname{Re}\left(n_{0+}^{0-}-n_{0+}^{+0}\right)\right] \\
&+\cos \left(\phi-\phi_{S}\right)\left[\cos (\phi+\varphi) \sqrt{\epsilon(1-\epsilon)} \operatorname{Re}\left(s_{0+}^{0+}-s_{0+}^{-0}\right)\right. \\
& \quad-\cos \varphi \sqrt{1-\epsilon^{2}} \operatorname{Re}\left(s_{++}^{0+}-s_{++}^{-0}\right) \\
&\left.\quad-\cos (\phi-\varphi) \sqrt{\epsilon(1-\epsilon)} \operatorname{Re}\left(s_{0+}^{0-}-s_{0+}^{+0}\right)\right] \\
& \quad \sin \left(\phi-\phi_{S}\right) \\
& \times \\
& \quad\left[\sin \phi \sqrt{\epsilon(1-\epsilon)} \operatorname{Re}\left(n_{0+}^{++}+n_{0+}^{--}\right)-\sin (\phi+2 \varphi) \sqrt{\epsilon(1-\epsilon)} \operatorname{Re} n_{0+}^{-+}\right. \\
& \quad+\sin (2 \varphi) \sqrt{1-\epsilon^{2}} \operatorname{Re} n_{++}^{-+} \\
&\left.\quad-\sin (\phi-2 \varphi) \sqrt{\epsilon(1-\epsilon)} \operatorname{Re} n_{0+}^{+-}\right] \\
&+\cos (\phi-\phi S)\left[\sqrt{1-\epsilon^{2}} \frac{1}{2}\left(s_{++}^{++}+s_{++}^{--}\right)\right. \\
& \quad
\end{aligned}
$$




\begin{tabular}{cccccccc}
\hline \hline \multicolumn{3}{c}{ unpolarized beam } & \multicolumn{4}{c}{ polarized beam } \\
$W_{U U}$ & $W_{U L}$ & $W_{U T}$ & $W_{L U}$ & $W_{L L}$ & $W_{L T}$ \\
$\operatorname{Re} u$ & $\operatorname{Im} l$ & $\operatorname{Im} n$ & $\operatorname{Im} s$ & $\operatorname{Im} u$ & $\operatorname{Re} l$ & $\operatorname{Re} n$ & $\operatorname{Re} s$ \\
15 & 14 & 16 & 14 & 8 & 10 & 8 & 10 \\
\hline \hline
\end{tabular}

Table 3: Number of linear combinations of spin density matrix elements describing the angular distribution of the cross section (4.5). The number of independent combinations for Re $u$ is one less than for $\operatorname{Im} n$ because of the relation (4.11).

$$
\begin{aligned}
& -\cos \phi \sqrt{\epsilon(1-\epsilon)} \operatorname{Re}\left(s_{0+}^{++}+s_{0+}^{--}\right)+\cos (\phi+2 \varphi) \sqrt{\epsilon(1-\epsilon)} \operatorname{Re} s_{0+}^{-+} \\
& -\cos (2 \varphi) \sqrt{1-\epsilon^{2}} \operatorname{Re} s_{++}^{-+} \\
& \left.+\cos (\phi-2 \varphi) \sqrt{\epsilon(1-\epsilon)} \operatorname{Re} s_{0+}^{+-}\right]
\end{aligned}
$$

for beam polarization. With obvious adjustments, the general structure discussed in points 1 to 3 above is found again for a transverse target. Note that the terms $u_{++}^{00}+\epsilon u_{00}^{00}$ and $u_{++}^{++}+u_{++}^{--}+2 \epsilon u_{00}^{++}$in the unpolarized coefficients $W_{U U}^{L L}$ and $W_{U U}^{T T}$ add up to 1 according to (4.11), whereas their counterparts $\operatorname{Im}\left(n_{++}^{00}+\epsilon n_{00}^{0} 0\right)$ and $\operatorname{Im}\left(n_{++}^{++}+n_{++}^{--}+2 \epsilon n_{00}^{++}\right)$in $W_{U T}^{L L}$ and $W_{U T}^{T T}$ are independent quantities. To keep the close similarity between the two cases we have not used (4.11) to simplify (4.10).

Since there are two independent transverse polarizations relative to the hadron plane (normal and sideways) we have a rather large number of terms with different angular dependence in (4.17) and (4.18). The single spin asymmetry $W_{U T}$ contains 16 terms with $\operatorname{Im} n$ and 14 terms with $\operatorname{Im} s$, whereas the double spin asymmetry $W_{L T}$ contains 8 terms with $\operatorname{Re} n$ and 10 terms with $\operatorname{Re} s$. Table 3 lists the number of independent linear combinations of spin density matrix elements describing the angular distribution for the different combinations of beam and target spin. For reasons discussed in Section 5 it is useful to consider the spin density matrices $n$ and $s$ separately. It is then natural to work in the basis of angular functions given by the product of $\sin \left(\phi-\phi_{S}\right)$ or $\cos \left(\phi-\phi_{S}\right)$ with $\sin (k \phi+m \varphi)$ or $\cos (k \phi+m \varphi)$. With the replacement rules (4.15) and (4.16) we obtain the combinations

$$
\begin{array}{r}
\sin \left(\phi-\phi_{S}\right) \cos (k \phi+m \varphi) \operatorname{Im} n+\cos \left(\phi-\phi_{S}\right) \sin (k \phi+m \varphi) \operatorname{Im} s, \\
-\sin \left(\phi-\phi_{S}\right) \sin (k \phi+m \varphi) \operatorname{Re} n+\cos \left(\phi-\phi_{S}\right) \cos (k \phi+m \varphi) \operatorname{Re} s
\end{array}
$$

in $W_{U T}$ and $W_{L T}$, respectively.

We conclude this section by giving the relation between our spin density matrix elements for an unpolarized target and those in the classical work [14] of Schilling and Wolf. We have

$$
\begin{aligned}
u_{++}^{00}+\epsilon u_{00}^{00} & =r_{00}^{04}, \\
\operatorname{Re}\left(u_{0+}^{0+}-u_{0+}^{-0}\right) & =\sqrt{2}\left(\operatorname{Im} r_{10}^{6}-\operatorname{Re} r_{10}^{5}\right), \\
u_{++}^{++}+u_{++}^{--}+2 \epsilon u_{00}^{++} & =1-r_{00}^{04} \\
u_{-+}^{-+} & =r_{1-1}^{1}-\operatorname{Im} r_{1-1}^{2},
\end{aligned}
$$




$$
\begin{aligned}
\operatorname{Re} u_{0+}^{00} & =-r_{00}^{5} / \sqrt{2}, \\
\operatorname{Re}\left(u_{++}^{0+}-u_{++}^{-0}+2 \epsilon u_{00}^{0+}\right) & =2 \operatorname{Re} r_{10}^{04}, \\
\operatorname{Re} u_{-+}^{0+} & =\operatorname{Re} r_{10}^{1}-\operatorname{Im} r_{10}^{2}, \\
\operatorname{Re}\left(u_{0+}^{0-}-u_{0+}^{+0}\right) & =\sqrt{2}\left(\operatorname{Im} r_{10}^{6}+\operatorname{Re} r_{10}^{5}\right), \\
\operatorname{Re}\left(u_{++}^{-+}+\epsilon u_{00}^{-+}\right) & =r_{1-1}^{04}, \\
\operatorname{Re} u_{-+}^{++} & =r_{11}^{1}, \\
\operatorname{Re}\left(u_{0+}^{++}+u_{0+}^{--}\right) & =-\sqrt{2} r_{11}^{5}, \\
\operatorname{Re} u_{0+}^{-+} & =\left(\operatorname{Im} r_{1-1}^{6}-r_{1-1}^{5}\right) / \sqrt{2}, \\
u_{-+}^{00} & =r_{00}^{1}, \\
\operatorname{Re} u_{-+}^{+0} & =\operatorname{Re} r_{10}^{1}+\operatorname{Im} r_{10}^{2}, \\
\operatorname{Re} u_{0+}^{+-} & =-\left(\operatorname{Im} r_{1-1}^{6}+r_{1-1}^{5}\right) / \sqrt{2}, \\
u_{-+}^{+-} & =r_{1-1}^{1}+\operatorname{Im} r_{1-1}^{2}
\end{aligned}
$$

and

$$
\begin{aligned}
\operatorname{Im}\left(u_{0+}^{0+}-u_{0+}^{-0}\right) & =\sqrt{2}\left(\operatorname{Im} r_{10}^{7}+\operatorname{Re} r_{10}^{8}\right), \\
\operatorname{Im} u_{0+}^{00} & =r_{00}^{8} / \sqrt{2}, \\
\operatorname{Im}\left(u_{++}^{0+}-u_{++}^{-0}\right) & =-2 \operatorname{Im} r_{10}^{3}, \\
\operatorname{Im}\left(u_{0+}^{0-}-u_{0+}^{+0}\right) & =\sqrt{2}\left(\operatorname{Im} r_{10}^{7}-\operatorname{Re} r_{10}^{8}\right), \\
\operatorname{Im} u_{++}^{-+} & =-\operatorname{Im} r_{1-1}^{3}, \\
\operatorname{Im}\left(u_{0+}^{++}+u_{0+}^{--}\right) & =\sqrt{2} r_{11}^{8}, \\
\operatorname{Im} u_{0+}^{-+} & =\left(\operatorname{Im} r_{1-1}^{7}+r_{1-1}^{8}\right) / \sqrt{2}, \\
\operatorname{Im} u_{0+}^{+-} & =-\left(\operatorname{Im} r_{1-1}^{7}-r_{1-1}^{8}\right) / \sqrt{2} .
\end{aligned}
$$

The lower indices in the matrix elements of Schilling and Wolf refer to the $\rho$ helicity and correspond to the upper indices of $u$ in our notation. Their upper indices correspond to a representation of the virtual photon spin density matrix which refers partly to circular and partly to linear polarization, whereas we use the helicity basis for the photon throughout. The consequences of approximate $s$-channel helicity conservation are more explicit in our notation: the relation $\operatorname{Im} r_{10}^{6} \approx-\operatorname{Re} r_{10}^{5}$ for instance corresponds to $\left|\operatorname{Re}\left(u_{0+}^{0+}-u_{0+}^{-0}\right)\right| \gg$ $\left|\operatorname{Re}\left(u_{0+}^{0-}-u_{0+}^{+0}\right)\right|$. Notice also that the simple relation between single-spin asymmetries and imaginary parts of spin density matrix elements discussed in point 3 above holds in the helicity basis but not for linear polarization.

We note that our phase convention (3.1) for the helicity states of the virtual photon differs from the one in [14] by a relative minus sign between transverse and longitudinal polarization, and that our normalization factors $N_{T}$ and $N_{L}$ in (3.5) differ from those in [14] by a factor of two. The combinations of helicity amplitudes corresponding to the spin density matrix elements in (4.20) and (4.21) should be compared according to

$$
\frac{1}{2}\left[\frac{1}{N_{T}+\epsilon N_{L}} \sum_{\sigma \lambda} T_{\mu \lambda}^{\nu \sigma}\left(T_{\mu^{\prime} \lambda}^{\nu^{\prime} \sigma}\right)^{*}\right]_{\text {here }}=\eta_{\mu \mu^{\prime}}\left[\frac{1}{N_{T}+\epsilon N_{L}} \sum_{\sigma \lambda} T_{\nu \sigma, \mu \lambda} T_{\nu^{\prime} \sigma, \mu^{\prime} \lambda}^{*}\right]_{[14]},
$$


where $\eta_{0 \pm}=\eta_{ \pm 0}=-1$ for the interference of transverse and longitudinal photon polarization, and $\eta_{\mu \mu^{\prime}}=+1$ in all other cases. ${ }^{4}$

\section{Natural and unnatural parity}

The exclusive process $\gamma^{*} p \rightarrow \rho p$ is described by eighteen independent helicity amplitudes, and we have already used approximate $s$-channel helicity conservation to establish a hierarchy among these amplitudes and the spin density matrix elements constructed from them. A further dynamical criterion to order these quantities is given by natural and unnatural parity exchange, which we shall now discuss.

Following [14] we define amplitudes $N$ for natural and $U$ unnatural parity exchange as linear combinations

$$
\begin{aligned}
& N_{\mu \lambda}^{\nu \sigma}=\frac{1}{2}\left[T_{\mu \lambda}^{\nu \sigma}+(-1)^{\nu-\mu} T_{-\mu \lambda}^{-\nu \sigma}\right]=\frac{1}{2}\left[T_{\mu \lambda}^{\nu \sigma}+(-1)^{\lambda-\sigma} T_{\mu-\lambda}^{\nu-\sigma}\right] \\
& U_{\mu \lambda}^{\nu \sigma}=\frac{1}{2}\left[T_{\mu \lambda}^{\nu \sigma}-(-1)^{\nu-\mu} T_{-\mu \lambda}^{-\nu \sigma}\right]=\frac{1}{2}\left[T_{\mu \lambda}^{\nu \sigma}-(-1)^{\lambda-\sigma} T_{\mu-\lambda}^{\nu-\sigma}\right] .
\end{aligned}
$$

With respect to the photon and meson helicity, the amplitudes $N$ have the same symmetry behavior as the amplitudes for $\gamma^{*} t \rightarrow \rho t$ on a spin-zero target $t$, whereas the corresponding relation for the amplitudes $U$ has an additional minus sign,

$$
N_{-\mu \lambda}^{-\nu \sigma}=(-1)^{\nu-\mu} N_{\mu \lambda}^{\nu \sigma}, \quad U_{-\mu \lambda}^{-\nu \sigma}=-(-1)^{\nu-\mu} U_{\mu \lambda}^{\nu \sigma}
$$

For the proton helicity we have relations $N_{\mu+}^{\nu+}=N_{\mu-}^{\nu-}$ and $N_{\mu-}^{\nu+}=-N_{\mu+}^{\nu-}$ for natural parity exchange, compared to $U_{\mu+}^{\nu+}=-U_{\mu-}^{\nu-}$ and $U_{\mu-}^{\nu+}=U_{\mu+}^{\nu-}$ for unnatural parity exchange. This symmetry behavior immediately implies that in a dynamical description using generalized parton distributions, amplitudes $N$ go with distributions $H$ and $E$, whereas amplitudes $U$ go with distributions $\tilde{H}$ and $\tilde{E}$. This is explicitly borne out in the calculation of [24]. Since $U_{0 \lambda}^{0 \sigma}=0$ according to (5.2), unnatural parity exchange amplitudes are power suppressed at large $Q^{2}$ and the leading-twist factorization theorem [2] only applies to the natural parity exchange amplitudes $N_{0 \lambda}^{0 \sigma}$. We remark that in the context of low-energy dynamics $t$-channel exchange of a pion plays a prominent role for unnatural parity exchange amplitudes, see e.g. [15]. This has a natural counterpart in the framework of generalized parton distributions, where pion exchange gives an essential contribution to the distribution $\tilde{E}$ in the isovector channel $[28,3,29]$.

\footnotetext{
${ }^{4}$ The correspondence in (4.20) to (4.22) is obtained from comparing our results (4.10) and (4.12) for the angular distribution with the ones in eqs. (92) and (92a) of [14], together with the relation between spin density matrix elements and helicity amplitudes specified in eq. (91) and Appendix A of [14]. We have not found an explicit specification of the phase convention for the virtual photon polarizations used in [14].
} 
For the spin density matrix elements one readily finds

$$
\begin{aligned}
& u_{\mu \mu^{\prime}}^{\nu \nu^{\prime}}=\left(N_{T}+\epsilon N_{L}\right)^{-1} \sum_{\sigma}\left[N_{\mu+}^{\nu \sigma}\left(N_{\mu^{\prime}+}^{\nu^{\prime} \sigma}\right)^{*}+U_{\mu+}^{\nu \sigma}\left(U_{\mu^{\prime}+}^{\nu^{\prime} \sigma}\right)^{*}\right], \\
& l_{\mu \mu^{\prime}}^{\nu \nu^{\prime}}=\left(N_{T}+\epsilon N_{L}\right)^{-1} \sum_{\sigma}\left[N_{\mu+}^{\nu \sigma}\left(U_{\mu^{\prime}+}^{\nu^{\prime} \sigma}\right)^{*}+U_{\mu+}^{\nu \sigma}\left(N_{\mu^{\prime}+}^{\nu^{\prime} \sigma}\right)^{*}\right], \\
& s_{\mu \mu^{\prime}}^{\nu \nu^{\prime}}=\left(N_{T}+\epsilon N_{L}\right)^{-1} \sum_{\sigma}\left[N_{\mu+}^{\nu \sigma}\left(U_{\mu^{\prime}-}^{\nu^{\prime} \sigma}\right)^{*}+U_{\mu+}^{\nu \sigma}\left(N_{\mu^{\prime}-}^{\nu^{\prime} \sigma}\right)^{*}\right], \\
& n_{\mu \mu^{\prime}}^{\nu \nu^{\prime}}=\left(N_{T}+\epsilon N_{L}\right)^{-1} \sum_{\sigma}\left[N_{\mu+}^{\nu \sigma}\left(N_{\mu^{\prime}-}^{\nu^{\prime} \sigma}\right)^{*}+U_{\mu+}^{\nu \sigma}\left(U_{\mu^{\prime}-}^{\nu^{\prime} \sigma}\right)^{*}\right] .
\end{aligned}
$$

The matrix elements $u$ and $n$ hence involve a product of two natural parity exchange amplitudes plus a product of two amplitudes for unnatural parity exchange, whereas $l$ and $s$ involve the interference between natural and unnatural parity exchange [15]. To the extent that amplitudes $U$ are smaller than their counterparts $N$, one can thus expect that matrix elements $l$ and $s$ are small compared with $u$ and $n$ for equal helicity indices. Exceptions to this guideline are possible since products $N_{\mu+}^{\nu \sigma}\left(N_{\mu^{\prime}+}^{\nu^{\prime} \sigma}\right)^{*}$ or $N_{\mu-}^{\nu \sigma}\left(N_{\mu^{\prime}+}^{\nu^{\prime} \sigma}\right)^{*}$ may have a small real or imaginary part due to the relative phase between the two amplitudes. If amplitudes $U$ are smaller than $N$, one can furthermore neglect the terms

$$
\begin{aligned}
& \tilde{u}_{\mu \mu^{\prime}}^{\nu \nu^{\prime}}=\left(N_{T}+\epsilon N_{L}\right)^{-1} \sum_{\sigma} U_{\mu+}^{\nu \sigma}\left(U_{\mu^{\prime}+}^{\nu^{\prime} \sigma}\right)^{*}, \\
& \tilde{n}_{\mu \mu^{\prime}}^{\nu \nu^{\prime}}=\left(N_{T}+\epsilon N_{L}\right)^{-1} \sum_{\sigma} U_{\mu+}^{\nu \sigma}\left(U_{\mu^{\prime}-}^{\nu^{\prime} \sigma}\right)^{*}
\end{aligned}
$$

involving unnatural parity exchange in the matrix elements $u$ and $n$. Using the relations

$$
(-1)^{\nu-\mu} u_{-\mu \mu^{\prime}}^{-\nu \nu^{\prime}}=u_{\mu \mu^{\prime}}^{\nu \nu^{\prime}}-2 \tilde{u}_{\mu \mu^{\prime}}^{\nu \nu^{\prime}}
$$

following from (5.2) and (5.3), we have in particular

$$
\begin{array}{ll}
-u_{-+}^{0+}=u_{++}^{0+}-2 \tilde{u}_{++}^{0+}, & u_{-+}^{-+}=u_{++}^{++}-2 \tilde{u}_{++}^{++}, \\
-u_{0+}^{-+}=u_{0+}^{++}-2 \tilde{u}_{0+}^{++}, & u_{-+}^{++}=u_{++}^{-+}-2 \tilde{u}_{++}^{-+} .
\end{array}
$$

This allows us to rewrite

$$
\begin{aligned}
W_{U U}^{L T}= & -\cos \varphi \operatorname{Re}\left(u_{++}^{0+}-u_{++}^{-0}+2 \epsilon u_{00}^{0+}\right)-\cos (2 \phi+\varphi) \epsilon \operatorname{Re}\left(u_{++}^{0+}-2 \tilde{u}_{++}^{0+}\right) \\
& +\ldots \cos (\phi+\varphi)+\ldots \cos (\phi-\varphi)+\ldots \cos (2 \phi-\varphi) \\
W_{U U}^{T T}= & \frac{1}{2}\left(u_{++}^{++}+u_{++}^{--}+2 \epsilon u_{00}^{++}\right)+\frac{1}{2} \cos (2 \phi+2 \varphi) \epsilon\left(u_{++}^{++}-2 \tilde{u}_{++}^{++}\right) \\
& -\cos \phi \sqrt{\epsilon(1+\epsilon)} \operatorname{Re}\left(u_{0+}^{++}+u_{0+}^{--}\right)-\cos (\phi+2 \varphi) \sqrt{\epsilon(1+\epsilon)} \operatorname{Re}\left(u_{0+}^{++}-2 \tilde{u}_{0+}^{++}\right) \\
& -\cos (2 \varphi) \operatorname{Re}\left(u_{++}^{-+}+\epsilon u_{00}^{-+}\right)-\cos (2 \phi) \epsilon \operatorname{Re}\left(u_{++}^{-+}-2 \tilde{u}_{++}^{-+}\right) \\
& +\ldots \cos (\phi-2 \varphi)+\ldots \cos (2 \phi-2 \varphi),
\end{aligned}
$$




$$
\begin{aligned}
W_{L U}^{T T}= & -\sin \phi \sqrt{\epsilon(1-\epsilon)} \operatorname{Im}\left(u_{0+}^{++}+u_{0+}^{--}\right)-\sin (\phi+2 \varphi) \sqrt{\epsilon(1-\epsilon)} \operatorname{Im}\left(u_{0+}^{++}-2 \tilde{u}_{0+}^{++}\right) \\
& +\ldots \sin (2 \varphi)+\ldots \sin (\phi-2 \varphi)
\end{aligned}
$$

where terms indicated by ... are the same as in the original expressions (4.10) and (4.12) and have not been repeated for brevity. We see that the coefficients of adjacent terms in (5.7) will be approximately equal to the extent that unnatural parity exchange is suppressed and $s$-channel helicity approximately conserved. This can be tested experimentally by measuring the angular distribution of the final-state particles.

The relations (5.6) and their counterparts for other index combinations can also be used to approximately isolate spin density matrix elements of particular interest. Consider as an example the leading-twist matrix element $u_{00}^{00}$, which in the angular distribution appears only in the combination $u_{++}^{00}+\epsilon u_{00}^{0} 0$, i.e. together with a matrix element that should be suppressed since it does not conserve $s$-channel helicity. If unnatural parity exchange is strongly suppressed, an even better approximation for $u_{00}^{00}$ can be obtained from the linear combination

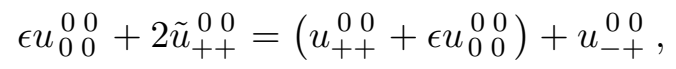

whose r.h.s. can be extracted from the angular distribution. Similarly, one can approximately isolate the matrix element $\operatorname{Re} u_{00}^{0+}$ in the combination

$$
\epsilon \operatorname{Re} u_{00}^{0+}+\operatorname{Re}\left(\tilde{u}_{++}^{0+}-\tilde{u}_{++}^{-0}\right)=\frac{1}{2}\left[\operatorname{Re}\left(u_{++}^{0+}-u_{++}^{-0}+2 \epsilon u_{00}^{0+}\right)+\operatorname{Re} u_{-+}^{0+}+\operatorname{Re} u_{-+}^{+0}\right] .
$$

Conversely, one can extract from the angular distribution the linear combinations

$$
\begin{aligned}
\tilde{u}_{++}^{++}+\tilde{u}_{--}^{++}+2 \epsilon \tilde{u}_{00}^{++}-2 \operatorname{Re} \tilde{u}_{-+}^{++}= & \frac{1}{2}\left(u_{++}^{++}+u_{++}^{--}+2 \epsilon u_{00}^{++}\right)-\frac{1}{2} u_{-+}^{-+}-\frac{1}{2} u_{-+}^{+-} \\
& +\operatorname{Re}\left(u_{++}^{-+}+\epsilon u_{00}^{-+}\right)-\operatorname{Re} u_{-+}^{++}, \\
\tilde{u}_{0+}^{++}+\tilde{u}_{0+}^{--}= & \frac{1}{2}\left(u_{0+}^{++}+u_{0+}^{--}\right)+\frac{1}{2} u_{0+}^{-+}+\frac{1}{2} u_{0+}^{+-},
\end{aligned}
$$

which only involve unnatural parity exchange. In a dynamical approach based on generalized parton distributions, these combinations are interesting because they isolate the polarized distributions $\tilde{H}$ and $\tilde{E}$ and in particular involve these distributions for gluons, which are very hard to access in any other process. ${ }^{5}$ The price to pay for this is that the corresponding amplitudes are power suppressed and cannot be calculated with the theoretical rigor provided by the leading-twist factorization theorem. On the other hand, phenomenological analysis indicates that a quantitative description of meson production at $Q^{2}$ of a few $\mathrm{GeV}^{2}$ requires the inclusion of power-suppressed effects also for the leading matrix element $u_{0}^{0} 00$.

The discussion of the matrix elements for transverse target polarization normal to the hadron plane proceeds in full analogy to the unpolarized case. With

$$
(-1)^{\nu-\mu} n_{-\mu \mu^{\prime}}^{-\nu \nu^{\prime}}=n_{\mu \mu^{\prime}}^{\nu \nu^{\prime}}-2 \tilde{n}_{\mu \mu^{\prime}}^{\nu \nu^{\prime}}
$$

\footnotetext{
${ }^{5}$ In contrast to their quark counterparts, $\tilde{H}^{g}$ and $\tilde{E}^{g}$ do not appear in pseudoscalar meson production at leading twist and leading order in $\alpha_{s}$, see e.g. Section 5.1.1 of [30].
} 
we have

$$
\begin{array}{ll}
-n_{-+}^{0+}=n_{++}^{0+}-2 \tilde{n}_{++}^{0+}, & n_{-+}^{-+}=n_{++}^{++}-2 \tilde{n}_{++}^{++}, \\
-n_{0+}^{-+}=n_{0+}^{++}-2 \tilde{n}_{0+}^{++}, & n_{-+}^{++}=n_{++}^{-+}-2 \tilde{n}_{++}^{-+}
\end{array}
$$

and can write

$$
\begin{aligned}
W_{U T}^{L T}= & \cos \left(\phi-\phi_{S}\right)[\ldots]+\sin \left(\phi-\phi_{S}\right) \\
\times & {\left[-\cos \varphi \operatorname{Im}\left(n_{++}^{0+}-n_{++}^{-0}+2 \epsilon n_{00}^{0+}\right)-\cos (2 \phi+\varphi) \epsilon \operatorname{Im}\left(n_{++}^{0+}-2 \tilde{n}_{++}^{0+}\right)\right.} \\
& +\ldots \cos (\phi+\varphi)+\ldots \cos (\phi-\varphi)+\ldots \cos (2 \phi-\varphi)] \\
W_{U T}^{T T}=\cos \left(\phi-\phi_{S}\right)[\ldots]+\sin \left(\phi-\phi_{S}\right) & \\
\times & {\left[\frac{1}{2} \operatorname{Im}\left(n_{++}^{++}+n_{++}^{--}+2 \epsilon n_{00}^{++}\right)+\frac{1}{2} \cos (2 \phi+2 \varphi) \epsilon \operatorname{Im}\left(n_{++}^{++}-2 \tilde{n}_{++}^{++}\right)\right.} \\
& -\cos \phi \sqrt{\epsilon(1+\epsilon)} \operatorname{Im}\left(n_{0+}^{++}+n_{0+}^{--}\right)-\cos (\phi+2 \varphi) \sqrt{\epsilon(1+\epsilon)} \operatorname{Im}\left(n_{0+}^{++}-2 \tilde{n}_{0+}^{++}\right) \\
& -\cos (2 \varphi) \operatorname{Im}\left(n_{++}^{-+}+\epsilon n_{00}^{-+}\right)-\cos (2 \phi) \epsilon \operatorname{Im}\left(n_{++}^{-+}-2 \tilde{n}_{++}^{-+}\right) \\
& +\ldots \cos (\phi-2 \varphi)+\ldots \cos (2 \phi-2 \varphi)] \\
W_{L T}^{T T}= & \cos \left(\phi-\phi \phi_{S}\right)[\ldots]+\sin \left(\phi-\phi_{S}\right) \\
\times & {\left[\sin \phi \sqrt{\epsilon(1-\epsilon)} \operatorname{Re}\left(n_{0+}^{++}+n_{0+}^{--}\right)+\sin (\phi+2 \varphi) \sqrt{\epsilon(1-\epsilon)} \operatorname{Re}\left(n_{0+}^{++} 2 \tilde{n}_{0+}^{++}\right)\right.} \\
& +\ldots \sin (2 \varphi)+\ldots \sin (\phi-2 \varphi)]
\end{aligned}
$$

where terms denoted by ... are as in the original expressions (4.17) and (4.18). Again, the coefficients of adjacent terms should be approximately equal to the extent that unnatural parity exchange is suppressed and $s$-channel helicity approximately conserved. The matrix elements $\operatorname{Im} n_{0}^{0} 0$ and $\operatorname{Im} n_{0}^{0+}$ can be approximately isolated in

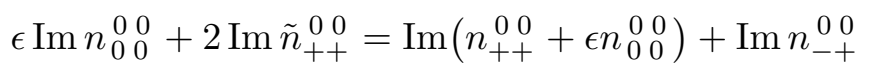

and

$$
\epsilon \operatorname{Im} n_{00}^{0+}+\operatorname{Im}\left(\tilde{n}_{++}^{0+}-\tilde{n}_{++}^{-0}\right)=\frac{1}{2}\left[\operatorname{Im}\left(n_{++}^{0+}-n_{++}^{-0}+2 \epsilon n_{00}^{0+}\right)+\operatorname{Im} n_{-+}^{0+}+\operatorname{Im} n_{-+}^{+0}\right] .
$$

In turn, the linear combinations

$$
\begin{aligned}
\operatorname{Im}\left(\tilde{n}_{++}^{++}+\tilde{n}_{--}^{++}+2 \epsilon \tilde{n}_{00}^{++}-2 \tilde{n}_{-+}^{++}\right)= & \frac{1}{2} \operatorname{Im}\left(n_{++}^{++}+n_{++}^{--}+2 \epsilon n_{00}^{++}\right)-\frac{1}{2} \operatorname{Im} n_{-+}^{-+}-\frac{1}{2} \operatorname{Im} n_{-+}^{+-} \\
& +\operatorname{Im}\left(n_{++}^{-+}+\epsilon n_{00}^{-+}\right)-\operatorname{Im} n_{-+}^{++} \\
\tilde{n}_{0+}^{++}+\tilde{n}_{0+}^{--}= & \frac{1}{2}\left(n_{0+}^{++}+n_{0+}^{--}\right)+\frac{1}{2} n_{0+}^{-+}+\frac{1}{2} n_{0+}^{+-}
\end{aligned}
$$

involve only unnatural parity exchange. 


\section{Positivity constraints}

From the definition (3.4) of the spin-density matrix elements one readily finds

$$
\sum_{\nu \mu \lambda} \sum_{\nu^{\prime} \mu^{\prime} \lambda^{\prime}} c_{\mu \lambda}^{\nu} \rho_{\mu \mu^{\prime}, \lambda \lambda^{\prime}}^{\nu \nu^{\prime}}\left(c_{\mu^{\prime} \lambda^{\prime}}^{\nu^{\prime}}\right)^{*}=\left(N_{T}+\epsilon N_{L}\right)^{-1} \sum_{\sigma}\left|\sum_{\nu \mu \lambda} c_{\mu \lambda}^{\nu} T_{\mu \lambda}^{\nu \sigma}\right|^{2} \geq 0
$$

for arbitrary complex numbers $c_{\mu \lambda}^{\nu}$. Hence $\rho_{\mu \mu^{\prime}, \lambda \lambda^{\prime}}^{\nu \nu^{\prime}}$ is a positive semidefinite matrix, with row indices specified by $\{\nu \mu \lambda\}$ and column indices by $\left\{\nu^{\prime} \mu^{\prime} \lambda^{\prime}\right\}$. This implies inequalities among the spin density matrix elements, which extend those given e.g. in [22, 27]. We do not attempt here to study the bounds following from positivity of the full $18 \times 18$ matrix $\rho_{\mu \mu^{\prime}, \lambda \lambda^{\prime}}^{\nu \nu^{\prime}}$, which is quite unwieldy. Instead, we consider the subset of matrix elements conserving $s$-channel helicity for the photon-meson transition and derive a number of simple inequalities, which may be useful in practice. Ordering the row and column indices as $\{+++\},\{00+\},\{--+\},\{++-\},\{00-\},\{---\}$, we have a positive semidefinite matrix $\mathrm{C}$, which can be written in block form as

$$
\mathrm{C}=\left(\begin{array}{ll}
\mathrm{A}_{+} & \mathrm{B}_{+} \\
\mathrm{B}_{-} & \mathrm{A}_{-}
\end{array}\right)
$$

with

$$
\mathrm{A}_{\eta}=\left(\begin{array}{ccc}
u_{++}^{++}+\eta l_{++}^{++} & \left(u_{0+}^{0+}+\eta l_{0+}^{0+}\right)^{*} & u_{-+}^{-+}-\eta l_{-+}^{-+} \\
u_{0+}^{0+}+\eta l_{0+}^{0+} & u_{00}^{00} & u_{0+}^{0+}-\eta l_{0+}^{0+} \\
u_{-+}^{-+}+\eta l_{-+}^{-+} & \left(u_{0+}^{0+}-\eta l_{0+}^{0+}\right)^{*} & u_{++}^{++}-\eta l_{++}^{++}
\end{array}\right)
$$

and

$$
\mathrm{B}_{\eta}=\left(\begin{array}{ccc}
s_{++}^{++}+\eta n_{++}^{++} & \left(s_{0+}^{0+}-\eta n_{0+}^{0+}\right)^{*} & -s_{-+}^{-+}+\eta n_{-+}^{-+} \\
s_{0+}^{0+}+\eta n_{0+}^{0+} & \eta n_{00}^{00} & -s_{0+}^{0+}+\eta n_{0+}^{0+} \\
s_{-+}^{-+}+\eta n_{-+}^{-+} & -\left(s_{0+}^{0+}+\eta n_{0+}^{0+}\right)^{*} & -s_{++}^{++}+\eta n_{++}^{++}
\end{array}\right),
$$

where $\eta= \pm 1$. Concentrating first on the matrix elements for an unpolarized or longitudinally polarized target, we find that the matrix $\mathrm{A}_{\eta}$ has eigenvalues whose expressions are very lengthy and therefore restrict our attention to $2 \times 2$ submatrices. The matrix obtained from the first and third rows and columns of $\mathrm{A}_{+}$has eigenvalues

$$
u_{++}^{++} \pm \sqrt{\left(u_{-+}^{-+}\right)^{2}+\left(l_{++}^{++}\right)^{2}+\left(\operatorname{Im} l_{-+}^{-+}\right)^{2}},
$$

whose positivity implies a bound

$$
\left(l_{++}^{++}\right)^{2}+\left(\operatorname{Im} l_{-+}^{-+}\right)^{2} \leq\left(u_{++}^{++}\right)^{2}-\left(u_{-+}^{-+}\right)^{2} .
$$

Similarly, the matrix obtained from the first and second and the matrix obtained from the second and third rows and columns of $\mathrm{A}_{+}$have respective eigenvalues

$$
\begin{aligned}
& \frac{1}{2}\left(u_{++}^{++}+l_{++}^{++}+u_{0}^{0} 0 \begin{array}{ll}
0 \\
0
\end{array}\right) \pm \frac{1}{2} \sqrt{\left(u_{++}^{++}+l_{++}^{++}-u_{0}^{0} 0\right)^{2}+4\left|u_{0+}^{0+}+l_{0+}^{0+}\right|^{2}}, \\
& \frac{1}{2}\left(u_{++}^{++}-l_{++}^{++}+u_{00}^{0} 0\right) \pm \frac{1}{2} \sqrt{\left(u_{++}^{++}-l_{++}^{++}-u_{0}^{0} 0\right)^{2}+4\left|u_{0+}^{0+}-l_{0+}^{0+}\right|^{2}},
\end{aligned}
$$


whose positivity gives bounds

$$
\begin{aligned}
& \left(\operatorname{Re} u_{0+}^{0+}+\operatorname{Re} l_{0+}^{0+}\right)^{2}+\left(\operatorname{Im} u_{0+}^{0+}+\operatorname{Im} l_{0+}^{0+}\right)^{2} \leq u_{00}^{00}\left(u_{++}^{++}+l_{++}^{++}\right), \\
& \left(\operatorname{Re} u_{0+}^{0+}-\operatorname{Re} l_{0+}^{0+}\right)^{2}+\left(\operatorname{Im} u_{0+}^{0+}-\operatorname{Im} l_{0+}^{0+}\right)^{2} \leq u_{00}^{00}\left(u_{++}^{++}-l_{++}^{++}\right) .
\end{aligned}
$$

A weaker condition is obtained by taking the sum of these two bounds,

$$
\left(\operatorname{Re} l_{0+}^{0+}\right)^{2}+\left(\operatorname{Im} l_{0+}^{0+}\right)^{2} \leq u_{00}^{00} u_{++}^{++}-\left(\operatorname{Re} u_{0+}^{0+}\right)^{2}-\left(\operatorname{Im} u_{0+}^{0+}\right)^{2} .
$$

The bounds (6.6) and (6.9) have right-hand sides involving only matrix elements accessible with an unpolarized target and constrain the matrix elements for longitudinal target polarization on their left-hand sides.

As a second example let us derive conditions which involve only matrix elements $u$ and $n$. To this end we consider the matrix

$$
\mathrm{C}^{\prime}=\frac{1}{2}\left(\mathrm{C}+\mathrm{D}^{\dagger} \mathrm{CD}\right)
$$

with

$$
\mathrm{D}=\left(\begin{array}{llllll}
0 & 0 & 1 & 0 & 0 & 0 \\
0 & 1 & 0 & 0 & 0 & 0 \\
1 & 0 & 0 & 0 & 0 & 0 \\
0 & 0 & 0 & 0 & 0 & 1 \\
0 & 0 & 0 & 0 & 1 & 0 \\
0 & 0 & 0 & 1 & 0 & 0
\end{array}\right)
$$

which is half the sum of the positive semidefinite matrices $\mathrm{C}$ and $\mathrm{D}^{\dagger} \mathrm{CD}$ and hence positive semidefinite itself. One readily finds that matrix elements $l$ and $s$ drop out in $\mathrm{C}^{\prime}$, which reads

$$
\mathrm{C}^{\prime}=\left(\begin{array}{cccccc}
u_{++}^{++} & \left(u_{0+}^{0+}\right)^{*} & u_{-+}^{-+} & n_{++}^{++} & -\left(n_{0+}^{0+}\right)^{*} & n_{-+}^{-+} \\
u_{0+}^{0+} & u_{00}^{00} & u_{0+}^{0+} & n_{0+}^{0+} & n_{00}^{00} & n_{0+}^{0+} \\
u_{-+}^{-+} & \left(u_{0+}^{0+}\right)^{*} & u_{++}^{++} & n_{-+}^{-+} & -\left(n_{0+}^{0+}\right)^{*} & n_{++}^{++} \\
-n_{++}^{++} & \left(n_{0+}^{0+}\right)^{*} & -n_{-+}^{-+} & u_{++}^{++} & \left(u_{0+}^{0+}\right)^{*} & u_{-+}^{-+} \\
-n_{0+}^{0+} & -n_{00}^{00} & -n_{0+}^{0+} & u_{0+}^{0+} & u_{00}^{00} & u_{0+}^{0+} \\
-n_{-+}^{-+} & \left(n_{0+}^{0+}\right)^{*} & -n_{++}^{++} & u_{-+}^{-+} & \left(u_{0+}^{0+}\right)^{*} & u_{++}^{++}
\end{array}\right) .
$$

This matrix has three eigenvalues

$$
\begin{aligned}
& u_{++}^{++}-u_{-+}^{-+}+\operatorname{Im} n_{++}^{++}-\operatorname{Im} n_{-+}^{-+}, \\
& \frac{1}{2}\left(u_{++}^{++}+u_{-+}^{-+}+\operatorname{Im} n_{++}^{++}+\operatorname{Im} n_{-+}^{-+}+u_{00}^{0} 00+\operatorname{Im} n_{0}^{0} 00\right) \\
& \pm \frac{1}{2} \sqrt{\left(u_{++}^{++}+u_{-+}^{-+}+\operatorname{Im} n_{++}^{++}+\operatorname{Im} n_{-+}^{-+}-u_{00}^{00}-\operatorname{Im} n_{00}^{0} 0\right)^{2}+8\left|u_{0+}^{0+}-i n_{0+}^{0+}\right|^{2}}
\end{aligned}
$$

and three further eigenvalues obtained by reversing the sign of all matrix elements $n$. Their positivity results in the bounds

$$
\left(\operatorname{Im} n_{++}^{++}-\operatorname{Im} n_{-+}^{-+}\right)^{2} \leq\left(u_{++}^{++}-u_{-+}^{-+}\right)^{2}
$$


and

$$
\begin{aligned}
& 2\left(\operatorname{Re} u_{0+}^{0+}+\operatorname{Im} n_{0+}^{0+}\right)^{2}+2\left(\operatorname{Im} u_{0+}^{0+}-\operatorname{Re} n_{0+}^{0+}\right)^{2} \\
& \leq\left(\begin{array}{ll}
u_{0}^{0} 0 & 0 \\
0 & \operatorname{Im} n_{00}^{0} 0
\end{array}\right)\left(u_{++}^{++}+u_{-+}^{-+}+\operatorname{Im} n_{++}^{++}+\operatorname{Im} n_{-+}^{-+}\right), \\
& 2\left(\operatorname{Re} u_{0+}^{0+}-\operatorname{Im} n_{0+}^{0+}\right)^{2}+2\left(\operatorname{Im} u_{0+}^{0+}+\operatorname{Re} n_{0+}^{0+}\right)^{2} \\
& \leq\left(\begin{array}{lll}
u_{0}^{0} 0 & 0
\end{array}-\operatorname{Im} n_{00}^{0} 0\right)\left(u_{++}^{++}+u_{-+}^{-+}-\operatorname{Im} n_{++}^{++}-\operatorname{Im} n_{-+}^{-+}\right) .
\end{aligned}
$$

Omitting the terms with $\operatorname{Im} u_{0+}^{0+}$ and $\operatorname{Re} n_{0+}^{0+}$, one obtains bounds involving only matrix elements that are accessible with an unpolarized lepton beam.

As we have seen in Section 4, s-channel helicity conserving matrix elements can be extracted from the angular distribution under the approximation that $s$-channel helicity changing transitions are suppressed. The bounds derived in this section may be used to check the consistency of this approximation.

\section{Mixing between transverse and longitudinal polarization}

So far we have discussed target polarization longitudinal or transverse to the virtual photon direction in the target rest frame, which is natural from the point of view of the stronginteraction dynamics. In an experimental setup one has however definite target polarization with respect to the lepton beam direction. The transformation from one polarization basis to the other is readily performed using the relations (2.3). For a target having longitudinal polarization $P_{L}$ with respect to the lepton beam one finds

$$
\begin{aligned}
& \frac{d \sigma}{d \phi d \varphi d(\cos \vartheta) d x_{B} d Q^{2} d t}=\frac{1}{2 \pi} \frac{d \sigma}{d x_{B} d Q^{2} d t} \\
& \quad \times\left(W_{U U}+P_{L}\left[\cos \theta_{\gamma} W_{U L}-\sin \theta_{\gamma} W_{U T}\left(\phi_{S}=0\right)\right]\right. \\
& \left.\quad+P_{\ell} W_{L U}+P_{\ell} P_{L}\left[\cos \theta_{\gamma} W_{L L}-\sin \theta_{\gamma} W_{L T}\left(\phi_{S}=0\right)\right]\right) .
\end{aligned}
$$

Note that in this case the azimuthal angle $\psi$ in (4.5) needs to be defined with respect to some fixed spatial direction in the target rest frame, rather than with respect to the (vanishing) transverse component of the target polarization relative to the lepton beam. We have integrated over this angle in (7.1) because the cross section does not depend on it.

For a target having transverse polarization $P_{T}$ with respect to the lepton beam one has

$$
\begin{gathered}
\frac{d \sigma}{d \phi_{S} d \phi d \varphi d(\cos \vartheta) d x_{B} d Q^{2} d t}=\frac{1}{(2 \pi)^{2}} \frac{d \sigma}{d x_{B} d Q^{2} d t} \frac{\cos \theta_{\gamma}}{1-\sin ^{2} \theta_{\gamma} \sin ^{2} \phi_{S}} \\
\times\left(W_{U U}+P_{T} \frac{\cos \theta_{\gamma} W_{U T}+\sin \theta_{\gamma} \cos \phi_{S} W_{U L}}{\left(1-\sin ^{2} \theta_{\gamma} \sin ^{2} \phi_{S}\right)^{1 / 2}}\right. \\
\left.+P_{\ell} W_{L U}+P_{\ell} P_{T} \frac{\cos \theta_{\gamma} W_{L T}+\sin \theta_{\gamma} \cos \phi_{S} W_{L L}}{\left(1-\sin ^{2} \theta_{\gamma} \sin ^{2} \phi_{S}\right)^{1 / 2}}\right) .
\end{gathered}
$$

The factor $\cos \theta_{\gamma} /\left(1-\sin ^{2} \theta_{\gamma} \sin ^{2} \phi_{S}\right)$ comes from the change of variables from $d \psi$ to $d \phi_{S}$ 
in the cross section. The relation between these two angles is readily obtained by setting $P_{L}=0$ in $(2.3)$ and given in [22].

It is a straightforward (if somewhat lengthy) exercise to insert our results (4.13), (4.14) and (4.17), (4.18) into (7.1) and (7.2) and to rewrite the expressions in terms of a suitable basis of functions depending on the azimuthal angles. Here we only give the combinations needed in (7.2) for a transversely polarized target and an unpolarized beam,

$$
\begin{aligned}
& \cos \theta_{\gamma} W_{U T}^{L L}\left(\phi_{S}, \phi\right)+\sin \theta_{\gamma} \cos \phi_{S} W_{U L}^{L L}(\phi) \\
&=\sin \left(\phi-\phi_{S}\right) {\left[\cos \theta_{\gamma} \operatorname{Im}\left(n_{++}^{00}+\epsilon n_{00}^{00}\right)-\sin \theta_{\gamma} \sqrt{\epsilon(1+\epsilon)} \operatorname{Im} l_{0+}^{00}\right.} \\
&\left.-\cos (2 \phi)\left\{\cos \theta_{\gamma} \epsilon \operatorname{Im} n_{-+}^{00}-\sin \theta_{\gamma} \sqrt{\epsilon(1+\epsilon)} \operatorname{Im} l_{0+}^{00}\right\}\right\} \\
&\left.-2 \cos \phi\left\{\cos \theta_{\gamma} \sqrt{\epsilon(1+\epsilon)} \operatorname{Im} n_{0+}^{00}+\frac{1}{4} \sin \theta_{\gamma} \epsilon \operatorname{Im} l_{-+}^{00}\right\}\right] \\
&+\cos \left(\phi-\phi_{S}\right) {\left[-\sin (2 \phi)\left\{\cos \theta_{\gamma} \epsilon \operatorname{Im} s_{-+}^{00}+\sin \theta_{\gamma} \sqrt{\epsilon(1+\epsilon)} \operatorname{Im} l_{0+}^{00}\right\}\right.} \\
&\left.-2 \sin \phi\left\{\cos \theta_{\gamma} \sqrt{\epsilon(1+\epsilon)} \operatorname{Im} s_{0+}^{00}+\frac{1}{4} \sin \theta_{\gamma} \epsilon \operatorname{Im} l_{-+}^{00}\right\}\right] \\
&-\frac{1}{2} \sin \theta_{\gamma} \sin \left(\phi_{S}+2 \phi\right) \epsilon \operatorname{Im} l_{-+}^{00},
\end{aligned}
$$

$\cos \theta_{\gamma} W_{U T}^{L T}\left(\phi_{S}, \phi, \varphi\right)+\sin \theta_{\gamma} \cos \phi_{S} W_{U L}^{L T}(\phi, \varphi)$

$$
\begin{gathered}
=\sin \left(\phi-\phi_{S}\right)\left[\operatorname { c o s } ( \phi + \varphi ) \left\{\cos \theta_{\gamma} \sqrt{\epsilon(1+\epsilon)} \operatorname{Im}\left(n_{0+}^{0+}-n_{0+}^{-0}\right)\right.\right. \\
\left.+\frac{1}{2} \sin \theta_{\gamma}\left[\operatorname{Im}\left(l_{++}^{0+}-l_{++}^{-0}+2 \epsilon l_{00}^{0+}\right)+\epsilon \operatorname{Im} l_{-+}^{0+}\right]\right\} \\
-\cos (\phi-\varphi)\left\{\cos \theta_{\gamma} \sqrt{\epsilon(1+\epsilon)} \operatorname{Im}\left(n_{0+}^{0-}-n_{0+}^{+0}\right)\right. \\
\left.+\frac{1}{2} \sin \theta_{\gamma}\left[\operatorname{Im}\left(l_{++}^{0+}-l_{++}^{-0}+2 \epsilon l_{00}^{0+}\right)-\epsilon \operatorname{Im} l_{-+}^{+0}\right]\right\} \\
+\cos (2 \phi+\varphi)\left\{\cos \theta_{\gamma} \epsilon \operatorname{Im} n_{-+}^{0+}-\frac{1}{2} \sin \theta_{\gamma} \sqrt{\epsilon(1+\epsilon)} \operatorname{Im}\left(l_{0+}^{0+}-l_{0+}^{-0}\right)\right\} \\
+\cos (2 \phi-\varphi)\left\{\cos \theta_{\gamma} \epsilon \operatorname{Im} n_{-+}^{+0}+\frac{1}{2} \sin \theta_{\gamma} \sqrt{\epsilon(1+\epsilon)} \operatorname{Im}\left(l_{0+}^{0-}-l_{0+}^{+0}\right)\right\} \\
-\cos \varphi\left\{\cos \theta_{\gamma} \operatorname{Im}\left(n_{++}^{0+}-n_{++}^{-0}+2 \epsilon n_{00}^{0+}\right)\right. \\
\left.\left.-\frac{1}{2} \sin \theta_{\gamma} \sqrt{\epsilon(1+\epsilon)}\left[\operatorname{Im}\left(l_{0+}^{0+}-l_{0+}^{-0}\right)-\operatorname{Im}\left(l_{0+}^{0-}-l_{0+}^{+0}\right)\right]\right\}\right] \\
+\cos \left(\phi-\phi_{S}\right)\left[\operatorname { s i n } ( \phi + \varphi ) \left\{\cos \theta_{\gamma} \sqrt{\epsilon(1+\epsilon)} \operatorname{Im}\left(s_{0+}^{0+}-s_{0+}^{-0}\right)\right.\right. \\
\left.-\frac{1}{2} \sin \theta_{\gamma}\left[\operatorname{Im}\left(l_{++}^{0+}-l_{++}^{-0}+2 \epsilon l_{00}^{0+}\right)-\epsilon \operatorname{Im} l_{-+}^{0+}\right]\right\}
\end{gathered}
$$




$$
\begin{gathered}
-\sin (\phi-\varphi)\left\{\cos \theta_{\gamma} \sqrt{\epsilon(1+\epsilon)} \operatorname{Im}\left(s_{0+}^{0-}-s_{0+}^{+0}\right)\right. \\
\left.-\frac{1}{2} \sin \theta_{\gamma}\left[\operatorname{Im}\left(l_{++}^{0+}-l_{++}^{-0}+2 \epsilon l_{00}^{0+}\right)+\epsilon \operatorname{Im} l_{-+}^{+0}\right]\right\} \\
+\sin (2 \phi+\varphi)\left\{\cos \theta_{\gamma} \epsilon \operatorname{Im} s_{-+}^{0+}+\frac{1}{2} \sin \theta_{\gamma} \sqrt{\epsilon(1+\epsilon)} \operatorname{Im}\left(l_{0+}^{0+}-l_{0+}^{-0}\right)\right\} \\
+\sin (2 \phi-\varphi)\left\{\cos \theta_{\gamma} \epsilon \operatorname{Im} s_{-+}^{+0}-\frac{1}{2} \sin \theta_{\gamma} \sqrt{\epsilon(1+\epsilon)} \operatorname{Im}\left(l_{0+}^{0-}-l_{0+}^{+0}\right)\right\} \\
-\sin \varphi\left\{\cos \theta_{\gamma} \operatorname{Im}\left(s_{++}^{0+}-s_{++}^{-0}+2 \epsilon s_{00}^{0+}\right)\right. \\
\left.\left.-\frac{1}{2} \sin \theta_{\gamma} \sqrt{\epsilon(1+\epsilon)}\left[\operatorname{Im}\left(l_{0+}^{0+}-l_{0+}^{-0}\right)+\operatorname{Im}\left(l_{0+}^{0-}-l_{0+}^{+0}\right)\right]\right\}\right] \\
+\frac{1}{2} \sin \theta_{\gamma}\left\{\sin \left(\phi_{S}+2 \phi+\varphi\right) \epsilon \operatorname{Im} l_{-+}^{0+}+\sin \left(\phi_{S}+2 \phi-\varphi\right) \epsilon \operatorname{Im} l_{-+}^{+0}\right\}
\end{gathered}
$$

$$
\begin{aligned}
& \cos \theta_{\gamma} W_{U T}^{T T}\left(\phi_{S}, \phi, \varphi\right)+\sin \theta_{\gamma} \cos \phi_{S} W_{U L}^{T T}(\phi, \varphi) \\
& =\sin \left(\phi-\phi_{S}\right)\left[\frac{1}{2} \cos \theta_{\gamma} \operatorname{Im}\left(n_{++}^{++}+n_{++}^{--}+2 \epsilon n_{00}^{++}\right)-\frac{1}{2} \sin \theta_{\gamma} \sqrt{\epsilon(1+\epsilon)} \operatorname{Im}\left(l_{0+}^{++}+l_{0+}^{--}\right)\right. \\
& -\cos (2 \phi)\left\{\cos \theta_{\gamma} \epsilon \operatorname{Im} n_{-+}^{++}-\frac{1}{2} \sin \theta_{\gamma} \sqrt{\epsilon(1+\epsilon)} \operatorname{Im}\left(l_{0+}^{++}+l_{0+}^{--}\right)\right\} \\
& -\cos \phi\left\{\cos \theta_{\gamma} \sqrt{\epsilon(1+\epsilon)} \operatorname{Im}\left(n_{0+}^{++}+n_{0+}^{--}\right)+\frac{1}{2} \sin \theta_{\gamma} \epsilon \operatorname{Im} l_{-+}^{++}\right\} \\
& +\frac{1}{2} \cos (2 \phi+2 \varphi)\left\{\cos \theta_{\gamma} \epsilon \operatorname{Im} n_{-+}^{-+}-\sin \theta_{\gamma} \sqrt{\epsilon(1+\epsilon)} \operatorname{Im} l_{0+}^{-+}\right\} \\
& +\frac{1}{2} \cos (2 \phi-2 \varphi)\left\{\cos \theta_{\gamma} \epsilon \operatorname{Im} n_{-+}^{+-}-\sin \theta_{\gamma} \sqrt{\epsilon(1+\epsilon)} \operatorname{Im} l_{0+}^{+-}\right\} \\
& -\cos (2 \varphi)\left\{\cos \theta_{\gamma} \operatorname{Im}\left(n_{++}^{-+}+\epsilon n_{00}^{-+}\right)-\frac{1}{2} \sin \theta_{\gamma} \sqrt{\epsilon(1+\epsilon)}\left[\operatorname{Im} l_{0+}^{-+}+\operatorname{Im} l_{0+}^{+-}\right]\right\} \\
& +\cos (\phi+2 \varphi)\left\{\cos \theta_{\gamma} \sqrt{\epsilon(1+\epsilon)} \operatorname{Im} n_{0+}^{-+}+\frac{1}{4} \sin \theta_{\gamma}\left[\epsilon \operatorname{Im} l_{-+}^{-+}+2 \operatorname{Im}\left(l_{++}^{-+}+\epsilon l_{00}^{-+}\right)\right]\right\} \\
& \left.+\cos (\phi-2 \varphi)\left\{\cos \theta_{\gamma} \sqrt{\epsilon(1+\epsilon)} \operatorname{Im} n_{0+}^{+-}+\frac{1}{4} \sin \theta_{\gamma}\left[\epsilon \operatorname{Im} l_{-+}^{+-}-2 \operatorname{Im}\left(l_{++}^{-+}+\epsilon l_{00}^{-+}\right)\right]\right\}\right] \\
& +\cos \left(\phi-\phi_{S}\right)\left[-\sin (2 \phi)\left\{\cos \theta_{\gamma} \epsilon \operatorname{Im} s_{-+}^{++}+\frac{1}{2} \sin \theta_{\gamma} \sqrt{\epsilon(1+\epsilon)} \operatorname{Im}\left(l_{0+}^{++}+l_{0+}^{--}\right)\right\}\right. \\
& -\sin \phi\left\{\cos \theta_{\gamma} \sqrt{\epsilon(1+\epsilon)} \operatorname{Im}\left(s_{0+}^{++}+s_{0+}^{--}\right)+\frac{1}{2} \sin \theta_{\gamma} \epsilon \operatorname{Im} l_{-+}^{++}\right\} \\
& +\frac{1}{2} \sin (2 \phi+2 \varphi)\left\{\cos \theta_{\gamma} \epsilon \operatorname{Im} s_{-+}^{-+}+\sin \theta_{\gamma} \sqrt{\epsilon(1+\epsilon)} \operatorname{Im} l_{0+}^{-+}\right\} \\
& +\frac{1}{2} \sin (2 \phi-2 \varphi)\left\{\cos \theta_{\gamma} \epsilon \operatorname{Im} s_{-+}^{+-}+\sin \theta_{\gamma} \sqrt{\epsilon(1+\epsilon)} \operatorname{Im} l_{0+}^{+-}\right\} \\
& -\sin (2 \varphi)\left\{\cos \theta_{\gamma} \operatorname{Im}\left(s_{++}^{-+}+\epsilon s_{00}^{-+}\right)-\frac{1}{2} \sin \theta_{\gamma} \sqrt{\epsilon(1+\epsilon)}\left[\operatorname{Im} l_{0+}^{-+}-\operatorname{Im} l_{0+}^{+-}\right]\right\}
\end{aligned}
$$




$$
\begin{aligned}
& \quad+\sin (\phi+2 \varphi)\left\{\cos \theta_{\gamma} \sqrt{\epsilon(1+\epsilon)} \operatorname{Im} s_{0+}^{-+}+\frac{1}{4} \sin \theta_{\gamma}\left[\epsilon \operatorname{Im} l_{-+}^{-+}-2 \operatorname{Im}\left(l_{++}^{-+}+\epsilon l_{00}^{-+}\right)\right]\right\} \\
& \left.+\sin (\phi-2 \varphi)\left\{\cos \theta_{\gamma} \sqrt{\epsilon(1+\epsilon)} \operatorname{Im} s_{0+}^{+-}+\frac{1}{4} \sin \theta_{\gamma}\left[\epsilon \operatorname{Im} l_{-+}^{+-}+2 \operatorname{Im}\left(l_{++}^{-+}+\epsilon l_{00}^{-+}\right)\right]\right\}\right] \\
& +\frac{1}{4} \sin \theta_{\gamma}\left\{\sin \left(\phi_{S}+2 \phi+2 \varphi\right) \epsilon \operatorname{Im} l_{-+}^{-+}+\sin \left(\phi_{S}+2 \phi-2 \varphi\right) \epsilon \operatorname{Im} l_{-+}^{+-}\right\} \\
& -\frac{1}{2} \sin \theta_{\gamma} \sin \left(\phi_{S}+2 \phi\right) \epsilon \operatorname{Im} l_{-+}^{++} .
\end{aligned}
$$

Compared with (4.17) and (4.18) we have changed the order of terms such that one readily sees which coefficients $\cos \theta_{\gamma} \operatorname{Im} n$ or $\cos \theta_{\gamma} \operatorname{Im} s$ receive an admixture from the same coefficients $\sin \theta_{\gamma} \operatorname{Im} l$. The terms in the last lines of (7.3) and (7.4) and in the last two lines of (7.5) involve only coefficients $\sin \theta_{\gamma} \operatorname{Im} l$. They come with an angular dependence which is absent for $\sin \theta_{\gamma}=0$, as is readily seen by rewriting

$$
\sin \left(\phi_{S}+2 \phi+m \varphi\right)=-\sin \left(\phi-\phi_{S}\right) \cos (3 \phi+m \varphi)+\cos \left(\phi-\phi_{S}\right) \sin (3 \phi+m \varphi) .
$$

We see in (7.3) to (7.5) that from the angular dependence of the cross section for transverse target polarization one can extract linear combinations of terms $\cos \theta_{\gamma} \operatorname{Im} n$ and $\sin \theta_{\gamma} \operatorname{Im} l$ or of $\cos \theta_{\gamma} \operatorname{Im} s$ and $\sin \theta_{\gamma} \operatorname{Im} l$. To separate these terms requires an additional measurement with longitudinal target polarization. ${ }^{6}$ The expressions (7.3) to (7.5) allow us to see for which terms the admixture of $\sin \theta_{\gamma} \operatorname{Im} l$ terms can be expected to be small, so that $\operatorname{Im} n$ and $\operatorname{Im} s$ may be determined with reasonable accuracy without such an additional measurement. Let us discuss a few examples.

1. The leading-twist matrix element $n_{0}^{0} 00$ appears in the linear combination

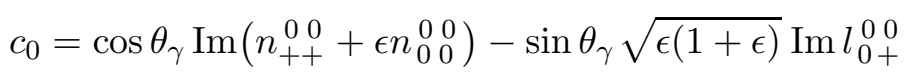

in (7.3) and thus has an admixture from $l_{0+}^{00}$, which involves one $s$-channel helicity changing amplitude. According to Section 5 this admixture is additionally suppressed if unnatural parity exchange is small compared with natural parity exchange. One may also add to $c_{0}$ the angular coefficient

$$
c_{1}=-\cos \theta_{\gamma} \epsilon \operatorname{Im} n_{-+}^{00}+\sin \theta_{\gamma} \sqrt{\epsilon(1+\epsilon)} \operatorname{Im} l_{0+}^{00}
$$

from (7.3), thus trading the admixture of $\sin \theta_{\gamma} l_{0+}^{00}$ for an admixture of $\cos \theta_{\gamma} n_{-+}^{00}$, which involves two $s$-channel helicity changing amplitudes (but lacks the relative factor $\tan \theta_{\gamma}$ and is not suppressed by unnatural parity exchange). We remark that the linear combination of matrix elements in (5.14) corresponds to $c_{0}-c_{1} / \epsilon$, where $l_{0+}^{00}$ does not drop out. Whether $c_{0}, c_{0}+c_{1}$ or $c_{0}-c_{1} / \epsilon$ gives the best approximation to $\cos \theta_{\gamma} \epsilon \operatorname{Im} n_{0}^{0} 00$ will thus depend on the detailed magnitude of the relevant terms. In practice one might for instance use the difference between these terms as a measure for the uncertainty of this approximation.

\footnotetext{
${ }^{6} \mathrm{~A}$ corresponding separation for semi-inclusive pion production $e p \rightarrow e \pi X$ has recently been performed in [31].
} 
2. The $s$-channel helicity conserving matrix elements $n_{0+}^{0+}$ in $(7.4)$ and $n_{++}^{++}, n_{-+}^{-+}$in (7.5) come together with terms involving at least one $s$-channel helicity changing amplitude. These admixtures should hence be negligible unless the corresponding $s$-channel helicity conserving matrix element is small itself. For $\operatorname{Im} n_{0+}^{0+}$ this may for instance happen because of the relative phase between the interfering amplitudes.

3. The matrix element $n_{0+}^{00}$ in (7.3) comes with an admixture from $l_{-+}^{00}$, which involves two $s$-channel helicity changing amplitudes and should hence again be suppressed. In addition, one can extract $\operatorname{Im} l_{-+}^{00}$ from the angular dependence itself, given the last term in (7.3). We remark that the unpolarized analog $u_{0+}^{00}$ of $n_{0+}^{00}$ has a real part which is experimentally seen to be nonzero $[17,19]$, providing evidence that $s$-channel helicity is not strictly conserved in electroproduction. (In the notation of Schilling and Wolf one has $r_{00}^{5}=-\sqrt{2} \operatorname{Re} u_{0+}^{0} 00$.)

4. The only $s$-channel helicity conserving matrix elements for sideways transverse target polarization in (7.3) to (7.5) are $s_{0+}^{0+}$ and $s_{-+}^{-+}$. They come together with terms involving at least one $s$-channel helicity changing amplitude, so that the situation is similar to the one in point 2. Note however that in the present case there is no additional suppression of the admixture terms due to unnatural parity exchange, since both $s$ and $l$ contain one unnatural parity exchange amplitude.

In these examples one thus has the favorable situation that the admixture from longitudinal polarization terms is probably small and in some cases may even be removed or traded for yet smaller terms. This does not always happen: the matrix elements $n_{-+}^{0+}$ and $s_{-+}^{0+}$ in (7.4) receive for instance an admixture from the $s$-channel helicity conserving term $l_{0+}^{0+}$, which may not be small itself, so that from the coefficients of $\sin \left(\phi-\phi_{S}\right) \cos (2 \phi+\varphi)$ or $\cos \left(\phi-\phi_{S}\right) \sin (2 \phi+\varphi)$ one cannot directly infer on the matrix elements $\operatorname{Im} n_{-+}^{0+}$ or $\operatorname{Im} s_{-+}^{0+}$. To make a more precise statement about their size one needs independent information on $\operatorname{Im} l_{0+}^{0+}$, for instance from the positivity bound (6.9).

\section{A note on non-resonant contributions}

So far we have treated the production of two pions in a two-step picture, where a $\rho$ is first produced in $e p \rightarrow e p \rho$ and then decays as $\rho \rightarrow \pi^{+} \pi^{-}$. For deriving the angular distribution and polarization dependence we have used that the pion pair is in the $L=1$ partial wave, as can be seen in (4.3). We did however not use the narrow-width approximation for the $\rho$ or make any assumption about its line shape. In fact, our results for the angular distribution can readily be used at any given invariant mass $m_{\pi \pi}$ of the pion pair, with the $e p$ cross sections on the left- and right-hand sides of (4.5) made differential in $m_{\pi \pi}$. The spin-density matrix $\rho_{\mu \mu^{\prime}, \lambda \lambda^{\prime}}^{\nu \nu^{\prime}}$ and its linear combinations $u, l, s, n$ then depend on $m_{\pi \pi}$ and refer not to $\gamma^{*} p \rightarrow \rho p$ but to $\gamma^{*} p \rightarrow \pi^{+} \pi^{-} p$ with $\pi^{+} \pi^{-}$in the $L=1$ partial wave. No explicit reference to the $\rho$ resonance needs to be made in this case.

The situation is more complicated if one considers other partial waves of the pion pair, which can arise from non-resonant production mechanisms. To describe a general $\pi^{+} \pi^{-}$ 
state, one should replace $\rho_{\mu \mu^{\prime}, \lambda \lambda^{\prime}}^{\nu \nu^{\prime}}$ with the spin-density matrix $\rho_{\mu \mu^{\prime}, \lambda \lambda^{\prime}}^{\nu \nu^{\prime}}$ for a pion pair with angular momentum $L$ in the amplitude and $L^{\prime}$ in the conjugate amplitude. One then has to take $Y_{L \nu}(\varphi, \vartheta) Y_{L^{\prime} \nu^{\prime}}^{*}(\varphi, \vartheta)$ instead of $Y_{1 \nu}(\varphi, \vartheta) Y_{1 \nu^{\prime}}^{*}(\varphi, \vartheta)$ in (4.3) and will obviously obtain a different angular dependence of the $e p$ cross section. The distribution in $\varphi$ and $\vartheta$ for a pion pair with $L=0,1,2$ has been discussed in [32].

It is quite simple to test for the presence of $L=0$ or $L=2$ partial waves in data by using discrete symmetry properties, and for $m_{\pi \pi}$ around the $\rho$ mass one can expect that partial waves with $L=3$ or higher are strongly phase space suppressed. Since even partial waves of the $\pi^{+} \pi^{-}$system have charge conjugation parity $C=+1$ and odd partial waves have $C=-1$, the interference of $L=1$ with $L=0$ or $L=2$ gives rise to terms in the angular distribution which are odd under interchange of the $\pi^{+}$and $\pi^{-}$momenta, i.e. under the replacement

$$
\vartheta \rightarrow \pi-\vartheta, \quad \varphi \rightarrow \varphi+\pi .
$$

Simple examples are an angular dependence like $\cos \vartheta$ or like an odd polynomial in $\cos \vartheta$. Corresponding observables provide a way to study the $L=0$ and $L=2$ partial waves as a "signal" interfering with the $\rho$ resonance "background" [33, 34]. This has been used in the experimental analysis [35], which did see such interference away from the $\rho$ resonance peak, whereas close to the peak the predominance of the $\rho$ was too strong to observe a significant contribution from any partial wave with $L \neq 1$. If on the other hand one is interested in a precise study of the $L=1$ component, one can eliminate its interference with even partial waves by symmetrizing the angular distribution according to (8.1). One is then left with contributions from $L=0$ and $L=2$ in both the amplitude and its conjugate, which should be very small around the $\rho$ peak.

\section{Summary}

We have expressed the fully differential cross section for exclusive $\rho$ production on a polarized nucleon in terms of spin density matrix element for the subprocess $\gamma^{*} p \rightarrow \rho p$. We work in the helicity basis for both $\gamma^{*}$ and $\rho$ and obtain very similar forms for the unpolarized and polarized parts of the cross sections, with the substitution rules (4.15) and (4.16). The terms for transverse target polarization normal to the hadron plane closely resemble those for an unpolarized target, and in both cases the number of independent spin density matrix elements is reduced if one neglects unnatural parity exchange compared with natural parity exchange. The spin density matrix elements for transverse target polarization in the hadron plane closely resemble those for a longitudinally polarized target, with both types of matrix elements involving the interference between natural and unnatural parity exchange. We have given simple positivity bounds which involve only matrix elements for an unpolarized target and either those for longitudinal target polarization or for transverse target polarization normal to the hadron plane. Furthermore, we have investigated the admixture of longitudinal target polarization relative to the virtual photon momentum for a target polarized transversely to the lepton beam. This admixture should be small for the spin density matrix elements which conserve $s$-channel helicity in the transition from 
$\gamma^{*}$ to $\rho$, but it may be important for $s$-channel helicity changing matrix elements. Finally, we have briefly discussed how the results obtained in this paper can be used and extended for analyzing the production of pion pairs not associated with the $\rho$ resonance.

\section{Acknowledgments}

It is a pleasure to thank my colleagues from HERMES for their interest in this work and for many discussions, especially A. Borissov, J. Dreschler, D. Hasch and A. Rostomyan. I also gratefully acknowledge helpful discussions with P. Kroll and A. Schäfer. This work is supported by the Helmholtz Association, contract number VH-NG-004.

\section{References}

[1] A. V. Radyushkin, Phys. Lett. B 385 (1996) 333 [hep-ph/9605431].

[2] J. C. Collins, L. Frankfurt and M. Strikman, Phys. Rev. D 56 (1997) 2982 [hep-ph/9611433].

[3] K. Goeke, M. V. Polyakov and M. Vanderhaeghen, Prog. Part. Nucl. Phys. 47 (2001) 401 [hep-ph/0106012].

[4] F. Ellinghaus, W.-D. Nowak, A. V. Vinnikov and Z. Ye, Eur. Phys. J. C 46 (2006) 729 [hep-ph/0506264].

[5] X. D. Ji, Phys. Rev. Lett. 78 (1997) 610 [hep-ph/9603249].

[6] M. Burkardt and G. Schnell, Phys. Rev. D 74 (2006) 013002 [hep-ph/0510249].

[7] M. Burkardt, Int. J. Mod. Phys. A 18 (2003) 173 [hep-ph/0207047].

[8] M. Diehl and A. V. Vinnikov, Phys. Lett. B 609 (2005) 286 [hep-ph/0412162].

[9] M. Diehl, W. Kugler, A. Schäfer and C. Weiss, Phys. Rev. D 72, 034034 (2005) [hep-ph/0506171].

[10] S. V. Goloskokov and P. Kroll, Eur. Phys. J. C 50 (2007) 829 [hep-ph/0611290].

[11] V. Uleshchenko and A. Szczurek, Acta Phys. Polon. B 33 (2002) 3299 [hep-ph/0207049].

[12] A. Airapetian et al. [HERMES Collab.], Phys. Rev. Lett. 94 (2005) 012002 [hep-ex/0408013].

[13] W. Vogelsang, hep-ph/0309295; A. Metz, hep-ph/0412156.

[14] K. Schilling and G. Wolf, Nucl. Phys. B 61 (1973) 381.

[15] H. Fraas, Annals Phys. 87 (1974) 417.

[16] M. R. Adams et al. [E665 Collab.], Z. Phys. C 74 (1997) 237.

[17] J. Breitweg et al. [ZEUS Collab.], Eur. Phys. J. C 12 (2000) 393 [hep-ex/9908026];

C. Adloff et al. [H1 Collab.], Eur. Phys. J. C 13 (2000) 371 [hep-ex/9902019].

[18] C. Adloff et al. [H1 Collab.], Phys. Lett. B 539 (2002) 25 [hep-ex/0203022].

[19] A. Borissov [HERMES Collab.], Proceedings of "Diffraction 06", PoS (DIFF2006) 014; B. Marianski [HERMES Collab.], Proceedings of "DIS 2006", World Scientific, Hackensack 2007, 255-258. 
[20] N. d'Hose [COMPASS Collab.], Proceedings of "DIS 2006", World Scientific, Hackensack 2007, 259-262.

[21] A. Bacchetta, U. D'Alesio, M. Diehl and C. A. Miller, Phys. Rev. D 70 (2004) 117504 [hep-ph/0410050].

[22] M. Diehl and S. Sapeta, Eur. Phys. J. C 41 (2005) 515 [hep-ph/0503023].

[23] P. Joos et al., Nucl. Phys. B 113 (1976) 53.

[24] S. V. Goloskokov and P. Kroll, Eur. Phys. J. C 42 (2005) 281 [hep-ph/0501242].

[25] M. Vanderhaeghen, P. A. M. Guichon and M. Guidal, Phys. Rev. D 60 (1999) 094017 [hep-ph/9905372].

[26] D. Yu. Ivanov and R. Kirschner, Phys. Rev. D 58 (1998) 114026 [hep-ph/9807324].

[27] T. Arens, O. Nachtmann, M. Diehl and P. V. Landshoff, Z. Phys. C 74 (1997) 651 [hep-ph/9605376].

[28] L. Mankiewicz, G. Piller and A. Radyushkin, Eur. Phys. J. C10 (1999) 307 [hep-ph/9812467].

[29] S. I. Ando, J. W. Chen and C. W. Kao, Phys. Rev. D 74 (2006) 094013 [hep-ph/0602200];

M. Diehl, A. Manashov and A. Schäfer, Eur. Phys. J. A31 (2007) 335 [hep-ph/0611101].

[30] M. Diehl, Phys. Rept. 388 (2003) 41 [hep-ph/0307382].

[31] A. Airapetian et al. [HERMES Collaboration], Phys. Lett. B 622 (2005) 14 [hep-ex/0505042].

[32] R. L. Sekulin, Nucl. Phys. B 56 (1973) 227.

[33] B. Lehmann-Dronke, A. Schäfer, M. V. Polyakov and K. Goeke, Phys. Rev. D 63 (2001) 114001 [hep-ph/0012108];

N. Warkentin, M. Diehl, D. Yu. Ivanov and A. Schäfer, Eur. Phys. J. A 32 (2007) 273 [hep-ph/0703148].

[34] P. Hägler, B. Pire, L. Szymanowski and O. V. Teryaev, Eur. Phys. J. C 26 (2002) 261 [hep-ph/0207224].

[35] A. Airapetian et al. [HERMES Collaboration], Phys. Lett. B 599 (2004) 212 [hep-ex/0406052]. 